\title{
"Can I Touch This?": Survey of Virtual Reality Interactions via Haptic Solutions
}

\author{
Revue de Littérature des Interactions en Réalité Virtuelle par le biais de Solutions Haptiques \\ Elodie Bouzbib \\ ISIR. Sorbonne Université \\ ISCD. Sorbonne Université \\ Paris, France \\ Gilles Bailly \\ Sinan Haliyo \\ ISIR. Sorbonne Université \\ Paris, France \\ Pascal Frey \\ ISCD. Sorbonne Université \\ Paris, France
}

\begin{abstract}
Haptic feedback has become crucial to enhance the user experiences in Virtual Reality (VR). This justifies the sudden burst of novel haptic solutions proposed these past years in the HCI community. This article is a survey of Virtual Reality interactions, relying on haptic devices. We propose two dimensions to describe and compare the current haptic solutions: their degree of physicality, as well as their degree of actuation. We depict a compromise between the user and the designer, highlighting how the range of required or proposed stimulation in VR is opposed to the haptic interfaces flexibility and their deployment in real-life use-cases. This paper (1) outlines the variety of haptic solutions and provides a novel perspective for analysing their associated interactions, (2) highlights the limits of the current evaluation criteria regarding these interactions, and finally (3) reflects the interaction, operation and conception potentials of "encountered-type of haptic devices".
\end{abstract}

\section{CCS CONCEPTS}

- Human-centered computing $\rightarrow$ Virtual reality; Haptic devices; Interaction design theory, concepts and paradigms.

\section{KEYWORDS}

haptics, Virtual Reality, human factors, haptic devices

\section{RÉSUMÉ}

Le retour haptique est devenu essentiel pour améliorer l'expérience utilisateur en Réalité Virtuelle (RV). C'est pourquoi nous observons une explosion du nombre de solutions haptiques proposées ces dernières années en IHM. Cet article est une revue de littérature des interactions en RV s'appuyant sur des dispositifs haptiques. Nous proposons deux dimensions pour décrire et comparer les solutions haptiques : leur degré de physicalité ainsi que leur degré de robotisation. Nous formulons un compromis utilisateur/concepteur, reflétant la variété des stimulations requises/proposées en RV, en opposition à la flexibilité des interfaces et leur déploiement en situation réelle. Ce travail (1) offre un panorama des solutions haptiques

Permission to make digital or hard copies of all or part of this work for personal or classroom use is granted without fee provided that copies are not made or distributed for profit or commercial advantage and that copies bear this notice and the full citation on the first page. Copyrights for components of this work owned by others than ACM must be honored. Abstracting with credit is permitted. To copy otherwise, or republish, to post on servers or to redistribute to lists, requires prior specific permission and/or a fee. Request permissions from permissions@acm.org.

IHM '20'21, April 13-16, 2021, Metz, France

(c) 2021 Association for Computing Machinery.

ACM ISBN 978-x-xxxx-xxxx-x/YY/MM ...\$15.00

https://doi.org/10.1145/XXXXXXXXX.XXXXXXXX en RV ainsi qu'un cadre d'analyse pour étudier les interactions associées, (2) souligne les limites des critères d'évaluation actuels pour ce type d'interactions, et finalement (3) reflète les potentiels interactionnel, opérationnel et conceptuel des interfaces haptiques "à contacts intermittents".

\section{MOTS-CLÉS}

haptique, Réalité Virtuelle, facteurs humains, dispositif haptique

\section{ACM Reference Format:}

Elodie Bouzbib, Gilles Bailly, Sinan Haliyo, and Pascal Frey. 2021. "Can I Touch This?": Survey of Virtual Reality Interactions via Haptic Solutions. In IHM '20'21: $32^{e}$ conférence Francophone sur l'Interaction Homme-Machine, April 13-16, 2021, Metz, France. ACM, New York, NY, USA, 16 pages. https: //doi.org/10.1145/XXXXXXXXX.XXXXXXXX

\section{INTRODUCTION}

In the last few years, the terms "Virtual Reality" and "Haptics" have been amongst the most quoted keywords in HCI conferences such as ACM CHI or ACM UIST. Indeed, Head-Mounted Displays (HMDs) are now affordable and provide high quality visual and audio feedback, but augmenting the experience by enhancing VR through the sense of touch (haptic feedback) has become a main challenge. A large variety of haptic solutions has currently been proposed, nonetheless they have highly different scopes, due to the wide range of haptic features. It is hence difficult to compare their similarities and differences and have a clear understanding of the design possibilities.

In this paper, we present a survey of existing haptic interactions in VR. We use the terms "haptic interactions" to emphasize the focus on the users actions, and to analyse how the "haptic devices" influence their behaviours in VR.

We provide a synthesis of existing research on haptic interactions in VR and depict, from the required haptic features stimulation and interaction opportunities, a design space discussing and classifying the associated haptic solutions according to two dimensions: their degree of physicality, i.e. their physical consistency and level of resemblance as to replicating an object, and their degree of actuation, i.e. whether they rely on a motor-based hardware implementation enabling autonomous displacements of the interface (eg changing its shape or position) (Table 1).

This design space is useful to characterize, classify and compare haptic interactions and the corresponding haptic solutions. We also propose two criteria, User experience and Conception costs, highlighting the implicit trade-offs between the quality of the user 
experience and the intricacy for the designer to implement these solutions. Both of the user's and designer's perspectives are hence considered in a novel framework to evaluate haptic interactions. Finally, we illustrate the utility of our design space by analyzing and comparing four haptic solutions. This analysis indicates that (1) the use of real props in a virtual environment benefits the user experience, but limits the interactions to the existing props available within the VR arena; (2) the use of robotised interfaces enables more various interactions; (3) combining them offers the best user experience/design cost trade-off; (4) current evaluation methods do not allow a fair representation and comparison of haptic solutions.

We hence propose guidelines to evaluate haptic interactions from both the user and designer perspectives. We also outline how intertwining interfaces can expand haptic opportunities, by conducting a deeper investigation on Robotic Graphics interfaces [101] . Indeed, in the quest of the Ultimate Display [147], these show (a) the largest variety of interactions, (b) the most reliable interfaces through their automation, and (c) the most natural interactions as they encounter the users at their positions of interest without further notice.

\section{BACKGROUND}

Surveys in Virtual Reality consider the technology itself and its limits [188, 191], or more specifically its use-case scenarios. VR is indeed used in industries [20,195], healthcare [103], or in gaming. In gaming, the concerns are mainly regarding the evaluation protocols [102], ie the presence [130] and its related questionnaires $[131,163]$. Surveys for instance compare the results whenever the questionnaires are asked in VR or in the real world $[9,116]$. The user behaviour in VR is also analysed, through gesture recognition [123] or system control techniques (eg menus) [25].

The research areas are coincidentally almost similar in haptics. Indeed, surveys analyse haptics themselves [164], haptic devices $[63,117,132,152]$ or examine the scenarios which benefit from a stimulation of the haptic cues. Haptics are used in telemanipulation [53], for training in the industry $[22,177]$ or for healthcare purposes [35, 118], or in gaming [78].

Finally, some surveys have been proposed at the intersection of VR and haptics and focus either on specific methods (pseudohaptic feedback) [96], technology according to stimulated haptic features (temperature, shape, skin stretch, pressure) [42, 169] or the motivations and applications of each haptic device category [168]. In contrast our survey outlines the variety of haptic interactions and technologies in VR and provides a framework to analyse them.

\section{SCOPE AND DEFINITIONS}

The scope of this article is to analyse how a single user interacts and is provided with believable haptic feedback in Virtual Reality [97]. We thus define the terms "virtual reality" and "haptics" and how they are related.

\subsection{Virtual Reality}

Virtual reality corresponds to a 3D artificial numeric environment in which users are immersed in. The environment can be projected onto a large screen, in a simulation platform for instance, or multiple ones, such as with CAVE technology (where the image is projected onto at least 3 distinct walls of a room-scale arena). In this survey, we consider an artificial reality [172] where users do not perceive their physical vicinity: the outside world is not noticeable and users are fully immersed through a head-mounted display (HMD). For instance, augmented reality (AR), where the physical environment is augmented with virtual artefacts, is out of our scope.

Through a Head Mounted Display (HMD), Virtual reality creates immersive experiences for the users. These are only limited by the designers' imagination, and are evaluated through presence. Presence is defined as the "subjective experience of being in one place, even when one is physically situated in another" [139, 175]. It quantifies the users' involvement and naturalness of interactions through control, sensory, distraction and realism factors. This heavily relies on the sensory input and output channels, however, as VR was mainly integrating audio and visual cues, quantifying the haptic contribution in an experience remains difficult.

\subsection{Haptics: Tactile vs Kinesthetic Perception}

Haptics is the general term for the sense of touch. They are a combination of two cues: tactile and kinesthetic. The tactile cues are developed through the skin, while the kinesthetic ones come from proprioception and are through the muscles and the tendons.

3.2.1 Tactile cues: The skin is composed of four types of mechanoreceptors [87]. The first ones, "Merkel nerve endings", transmit mechanical pressure, position and shapes or edges. They are stimulated whilst reading Braille for instance. The second ones, "Ruffini corpuscle end-organ", are sensitive to skin stretch and provide both pressure and slippage information. The third ones are the "Pacinian corpuscles", which are sensitive to vibration and pressure. The last ones, "Meissner's corpuscles", are highly sensitive and provide light touch and vibrations information. It also contains thermoreceptors, which transmit information about temperature: the Ruffini endings respond to warmth, while the Krause ones detect cold. Through tactile cues, the human can hence feel shapes or edges, pressure, vibrations or temperature changes.

3.2.2 Kinesthetic cues: The kinesthetic cues rely on proprioception, ie the perception and the awareness of our own body parts positions and movements. Mechanoreceptors into the muscles, the "spindles", communicate to the nervous system information the forces muscle generate, as well as their length change [77]. The primary type of spindle is sensitive to the velocity and acceleration of a muscle contraction or limb movement, while the second type provides information about static muscle length or limb positions. Kinesthetic cues hence allow to feel forces, as well as perceiving weights or inertia.

\subsection{VR \& Haptics}

Whenever we touch or manipulate an object, the combination of these two previous cues allows to understand its material, but also its shape and the constraints it implies to the user. On the one side, adding physical presence [92] through haptic feedback in VR enhances the users' immersion, even at an emotional and physiological scale: the heart rate of a user can literally increase 
with the use of haptics through real objects [73]. Haptics are also required for interacting with the environment: the user needs to control the changes in the environment [66] and to be aware of the modifications he physically has made (eg moving virtual objects, pushing a button). On the other side, haptics can benefit from VR. For instance, Lécuyer et al. leverage the users vision and analyse how it affects their haptic feedback [96]. This approach, "pseudohaptic feedback", tricks the users' perception into feeling virtual objects' stiffness, texture, mass. Many more haptic features can be stimulated, such as temperature, shape, skin stretch, pressure.

\section{ANALYZING HAPTIC INTERACTIONS}

The main objective of this survey is to provide analytical tools to evaluate and compare haptic interactions.

\subsection{Design space}

We propose a two-dimension framework to discuss and classify haptic solutions in VR (see Table 1).

The first dimension is their degree of physicality, ie how the haptic perception is tangible/physically consistent/resembling with the virtual objects. This dimension is drawn as a continuum, from "no physicality" to "real objects" (see Figure 2). We find that this continuum can be discretised as a two-category section: whether they use real objects or not.

The second orthogonal dimension is their degree of actuation, ie whether haptic solutions rely on a motor-based hardware implementation enabling autonomous displacements (eg enabling to change its shape, position etc).

\subsection{Analysis criteria}

We consider two main criteria to analyse haptic interactions in VR. They cover both the user and designer perspectives.

The User experience is the first criterion and includes two aspects: interaction opportunities and visuo-haptic consistency/discrepancy. Interaction opportunities represent to which extent haptic solutions allow users to interact/act (e.g navigate, explore, manipulate) in a VR scene as opposed as in the real world. Visuo-haptic consistency/discrepancy refers to the tactile and kinesthetic perceptual rendering of these interactions. These two subcriteria are complementary focusing on both action and perception.

The second criterion is the conception cost, i.e. the challenges Designers should address when designing haptic interactions. We distinguish implementation and operation costs. Implementation costs include several technical aspects related to the acceptability of a haptic solution such as safety, robustness and ease-of-use [42]. Operation costs include the financial and human costs required to deploy these technologies.

\subsection{Application}

We rely on this design space and criteria to highlight and understand the trade-offs between the user's interactions opportunities in VR, and the designers' challenges in conception. This survey offers a novel perspective for researchers to study haptic interactions in VR. It can be used to compare and analytically evaluate existing haptic interactions. For a given application, designers can evaluate the most adapted haptic interaction. For a given technique,

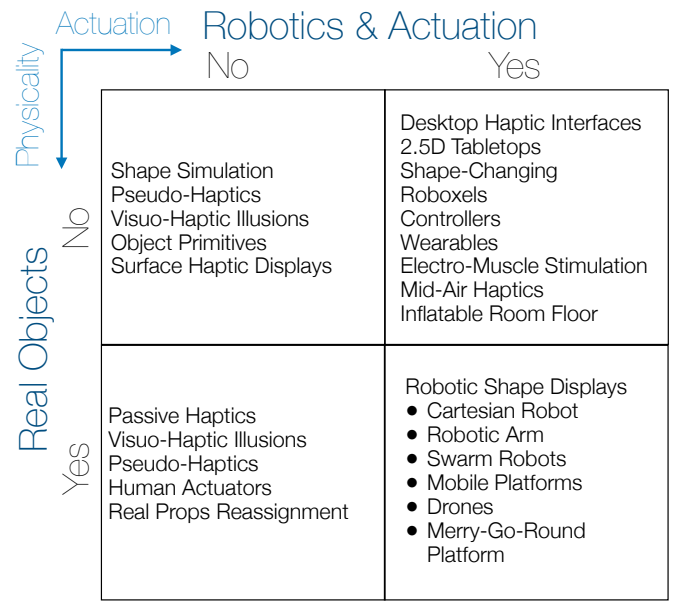

Table 1: We propose two dimensions to classify current technologies: their degrees of physicality and actuation.

they can evaluate a haptic solution depending on their needs (tasks, workspace, use-cases etc).

We first discuss haptic interactions from the User perspective (Section 5 - Interaction opportunities, Section 6 - Visuo-Haptic Consistency/Discrepancy). We then adopt the designer perspective in Section 7. We use our design space on Sections 6 and 7, which emphasize haptic solutions.

\section{INTERACTION OPPORTUNITIES}

In the real world, users move freely without constraints, pick any object of their environment and then interact with their bare-hands. They also can be interacted with, from the environment (wind, unexpected obstacles) or from other users, for instance to catch their attention or to lead them somewhere. A natural environment also naturally physically constrains users through their entire body.

In this section, we discuss the interaction opportunities in VR and the methods available to provide them. In particular, we discuss them through four main tasks: navigation, exploration, manipulation and edition.

\subsection{Navigation}

We qualify a navigation task as the exploration of the environment through the vision and the ability to navigate through it via the users displacements. We identify three main techniques to navigate in VR. The two firsts rely on controllers and push buttons, where the users do not necessary physically move. The last one is more natural as it allows the users to walk in the VR arena.

5.1.1 Panning: With grounded desktop haptic solutions, such as the Virtuose [62], users need to push a button to clutch the devices and hence move within the environment.

5.1.2 Point \& Teleport: With ungrounded solutions, such as controllers, the common technique is teleportation. Users point their controllers [14] to predetermined teleportation target areas, and are displaced in position but also in orientation [51] (Figure 1 - 1). 


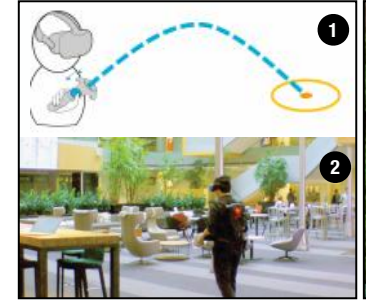

Navigation

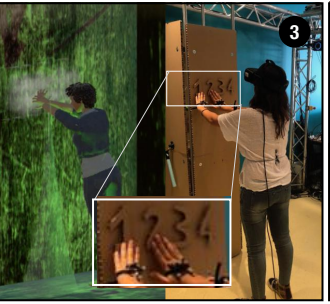

Exploration

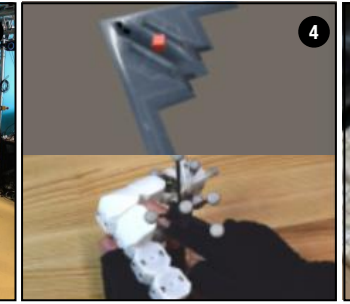

Manipulation

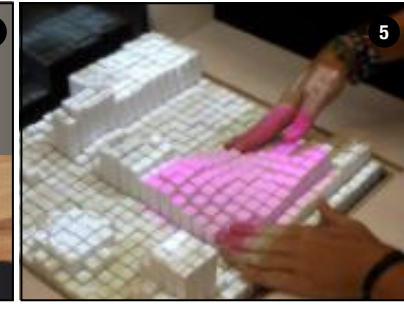

Edition

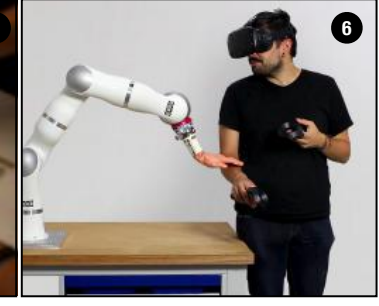

Being Interacted With

Figure 1: Tasks in VR: (1) Navigation through Point \& Teleport [51]; (2) Navigation through a building, using redirection [27]; (3) Exploration with Bare-Hands: A user finds an invisible haptic code [24]; (4) Manipulation: Haptic proxies rearrange themselves to form a plane the user can manipulate [190]; (5) Edition: the user changes the shape of a haptic $2.5 \mathrm{D}$ tabletop [105]; (6) The user is interacted with by a robotic arm to feel emotions [155].

5.1.3 Real Walking: Real walking in VR, "perambulation", has shown the best immersion and presence results $[142,162]$ because it relies on proprioception and kinesthetic feedback through the legs and gait awareness. Nonetheless, VR arenas are not infinite and HMD have a limited tracking space, hence methods need to be developed for the user to be able to move to any location of interest. One approach is to mount the previously discussed grounded desktop haptic solutions over mobile $[49,88,89,106,111,126]$ or wearable [17] interfaces. Users however still have to continuously maintain the handle in their palm. Other interfaces hence allow for freehands Room-Scale VR [24, 170, 181]. For the users to perambulate in an infinite workspace, the virtual environment can also visually be warped for the users to unconsciously modify their trajectory or avoid obstacles $[27,119,179]$ (Figure 1 - 2). This infinite redirection can also be provided from Electro-Muscle Stimulation (EMS) on the users' legs [12], with wearable electrodes. The user can also wear actuated stilts to perceive staircases [129] or a vibrating shoe to perceive virtual materials [145]. To remain unencumbered from these wearable techniques, the VR arena can also include robotised techniques: users can for instance walk on treadmills $[50,166]$, or on movable platforms that encounter their feet $[74,75]$.

\subsection{Hand Interactions}

In the real world, bare-hands interaction is important to execute everyday tasks (exploration, manipulation, edition). However, in VR, users commonly have to hold controllers, wearables or handles, which create a discrepancy between what the users feel and see [182]. These exploit the God-object principle [194], as opposed to bare-hands Real-touch interactions.

5.2.1 God-Object: The controller is considered as a continuity of the users' hands, represented by a proxy that does not undergo physics or rigid collisions, and is attached to a complementary rigid object with a spring-damper model. This latter hence moves along with the proxy, but is constrained by the environment. Whenever it does collide with an object of interest, the users perceive the previous spring-damper stiffness through kinesthetic feedback. Users hence interact though a proxy, like a desktop mouse, which position is not co-located with the users' vision. Bare-hands interactions are not necessarily needed depending on the use-cases. For instance, in healthcare and surgery training, users are more likely to interact with a tool, such as a scalpel or a clamp. Continuously holding the god-object is hence not a constrain, however the co-location of vision and haptics is recommended [109].

5.2.2 Real Touch: In other scenarios, such as gaming, industry or tool training $[143,174]$, using the appropriate tools through props and real objects is more natural. The users however need to be able to reach them whenever required. Some interfaces (e.g. Robotic Graphics; see Section 7.3) are hence developed in these regards, to encounter the users whenever they feel like interacting.

\subsection{Exploration}

As opposed to the previous definition of "navigation", based on vision cues, an "exploration" task consists in the ability to touch the environment and understand its constraints. Exploring thoroughly an environment in VR can be done through different haptic features, and can improve the users depth perception [98] or distances to an object. The different methods for exploring the environment are detailed in Section 6.

Whenever a user is exploring the environment, shapes or textures are felt through his body displacements. He needs to move for his skin to stretch (through tactile cues) or his muscles to contract (through kinesthetic cues).

5.3.1 Through Tactile cues: Whenever real props or material patches are available, users can naturally interact with their fingertips to feel different materials [11, 41], textures [19, 93], temperatures [192] or to feel shapes and patterns through their bare-hands $[24,30]$ (Figure 1 - 3). When no physicality is available, a stimulation can still be performed. As seen in Surface haptic displays [18], vibrations between 80 to $400 \mathrm{~Hz}$ are felt through the skin, hence users perceive stickiness, smoothness, pleasure, vibration or friction, and for instance explore a 3D terrain or volumetric data [137]. Vibrations can then be combined with auditory and vision cues to render collisions in VR [23].

5.3.2 Through Kinesthetic cues: Exploring the environment can also be done through kinesthetic cues: the users can literally be physically constrained to feel a wall, using electro-muscle stimulation (EMS) for instance [95]. With the god-object principle, users can also explore the environments' constraints through forcefeedback. In this configuration, the users' arms are constrained 
by haptic desktop interfaces, providing strong enough forces to simulate a physical collision and discriminate shapes.

\subsection{Manipulation}

A manipulation task is performed whenever modifying the position and orientation of an object.

5.4.1 Direct Manipulation: In VR, we distinguish the direct manipulation [26], "the ability for a user to control objects in a virtual environment in a direct and natural way, much as objects are manipulated in the real world" from pointing/selecting an object with controllers. A direct manipulation relies on the ability to hold an object with kinesthetic feedback, feel its weight [67, 95, 124, 134, 186, 187], shape $[48,85,146]$, and constrains from the virtual environment, for instance when making objects interact with each other [24]. Changing a virtual object position or orientation can be used as an input in the virtual environment: in [190] for instance, the user modifies a light intensity by moving a handle prop in the real environment. By transposing [94] in VR, an object could even communicate its dynamic use to the user.

5.4.2 Pseudo-Haptic Manipulation: Leveraging vision over haptics allows to move an object with different friction, weights or force perceptions $[115,120,121,125]$. For instance, visually reducing the speed of a virtual prop displacement leads to an increase in the users' forces to move it, modifying their friction/weight perceptions.

\subsection{Edition}

We qualify an Edition task as a modification of an object property, other than its orientation or position (for example through its scale [176] or shape).

5.5.1 Physical Edition: Editing an interface in VR requires it to be fully equipped with sensors. With wearables for instance, the hand phalanges positions are known, and can be tightly linked with an object property [165]. Knowing their own position, modular interfaces can be rearranged to provide stretching or bending tasks [46], or be pushed on with a tool to reduce in size [154].

Shape-changing interfaces have been developed to dynamically modify material properties [105] (Figure 1 - 5) or augment the interactions in Augmented Reality (AR) [91], however these techniques only consider HMDs and VR as future work directions.

These interfaces are relevant as 2.5D tabletops are already used in VR. Physically editing the virtual world through them could be implemented in a near future, by intertwining these interfaces with 3D modelling techniques [38].

5.5.2 Pseudo-Haptic Edition: The difficulty behind changing a real object property is to track it in real-time. This is why pseudotechniques are relevant: they visually change the object properties such as their shape [7], compliance [90, 136], or their bending curvature [68] without physically editing the object.

\subsection{Scenario-based Interactions}

In the real world, humans are free to interact with any object without further notice. In this regard, common controllers enable interactions with any object through pointing, but they display a high visuo-haptic discrepancy. In more advanced haptically rendered
Virtual environments, users are often constrained to scenario-based interactions: only a few interactable objects are available, accordingly with the scenario's progress.

The greater the virtual:physical haptic consistency, the harder it is to enhance non-deterministic scenarios, where the user is free to interact with any object with no regards to the scenario's progress. High quality haptic rendering in non-deterministic scenarios can be achieved through three methods: (a) numerous objects and primitives are available for interactions [69]; (b) the users' intentions are to be predicted prior to interaction to make it occur $[24,30]$; (c) props modify their own topology to match the users expected haptic rendering [138].

\subsection{Environment-Initiated Interactions}

In both real and virtual environments with tangible interfaces, users usually are the decision makers and get to choose their points of contact during the next interaction. However, users themselves can be considered as tangible interfaces: uncontrolled interactions, such as being touched by a colleague, or feeling a temperature change in the environment [133, 192], are part of everyday interactions that can be transposed in Virtual Reality. Replicating a social touch interaction in VR for instance increases presence [71] or invokes emotions [155].

This type of interactions are recurrent in sports simulations, where the user is undergoing forces from his environment and perceiving impacts (jumping into space [58], shooting a soccer ball [167], goalkeeping in a soccer game [157], paragliding [180], intercepting a volleyball [60], flying [29]).

These interactions are involving multiple force types: tension, traction, reaction, resistance, impact that help enhancing the user experience in VR [170]. These can be strong enough to even lead the user through forces [24].

\subsection{Whole-Body Involvement}

All the previous subsections evoke interactions that mainly involve the hands or the fingers. This paradigm is revoked in [193]: a user should be able to choose his posture. This is currently only enabled in room-scale VR applications, where users experience sitting, standing, climbing or crouching $[24,36,148,154]$ and interact with their whole-body.

\section{VISUO-HAPTIC CONSISTENCY/DISCREPANCY}

Visuo-Haptic Consistency is the second aspect of the user experience. We exploit the dimension degree of physicality of our design space (Table 1) to discuss the different haptic solutions. In particular, we distinguish whether these solutions use real objects (exploiting real objects) or not (simulating objects).

\subsection{Simulating Objects}

Object properties that need to be simulated are their shape, texture, temperature, weight.

6.1.1 No Physicality, (Figure 2 - 1). Currently, grounded haptic devices such as the Virtuose [62] or the PHaNToM [100] simulate objects through their shapes (Figure $2-1$ ). The rendering is only 


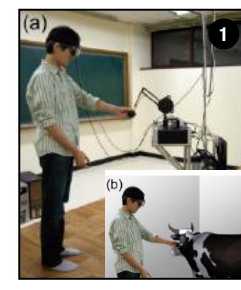

No Physicality

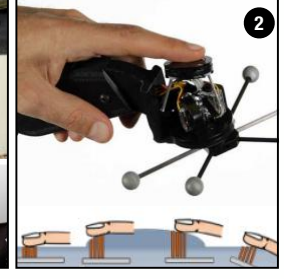

Shape Simulation

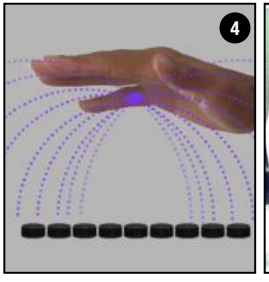

Object Primitives

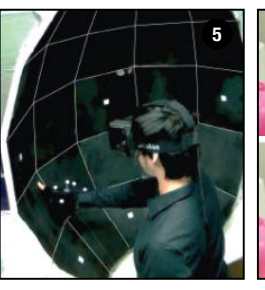

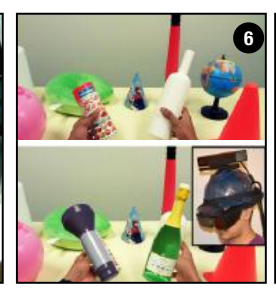

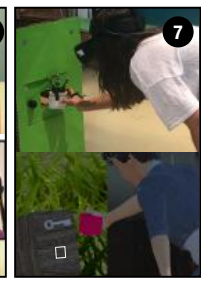

Real Objects

Figure 2: Degree of physicality continuum in VR.(1) Haptic desktop devices enable to explore the environment through a handle [89] with the god-object principle; (2) A controller [19] or (3) a wearable [45] simulate objects for exploration tasks; (4) Mid-air technology [117] create vibrations through the user's hand to simulate an object; (5) Passive proxies are oriented for the user to feel objects' primitives with their hands [30]; (6) Objects from the environment are assigned to virtual props with the same primitives [69]; (7) Real objects or passive props can be manipulated and interacted with each other [24].

done through kinesthetic feedback via a proxy. Conceptually, the ideal link between the users and this proxy is a massless, infinitely rigid stick, which would be an equivalent to moving the proxy directly $[63,127]$. These solutions only provide stimulation at the hand-scale, with no regards to the rest of the body.

6.1.2 Shape Simulation, (Figure 2 - 2-3-4). In the same regard, gloves or controllers provide some physicality (Figure 2 - 2-3). Gloves or exoskeletons literally constrain the users hands for simulating shapes $[2,6,8,10,32,33,45,57,104,114,158]$, or stimulate other haptic features such as stiffness, friction [165] or slippage [156]. These can be extended to overall body suits for users to feel impacts or even temperature changes $[3,37]$, or even intertwined with grounded devices to extend their use-cases [141].

Customised controllers are currently designed to be either stimulating the palm $[39,146,185]$ (Figure $3-1,2$ ), or held in the palm while providing haptic feedback on the fingertips. For instance, [173] proposes interchangeable haptic wheels with different textures or shapes, while [19] enables textures and shapes and [90] displays compliance changes. In these configurations, users hold a single controller, however bi-manual interactions can be created by combining two controllers. Their link transmits kinesthetic feedback,
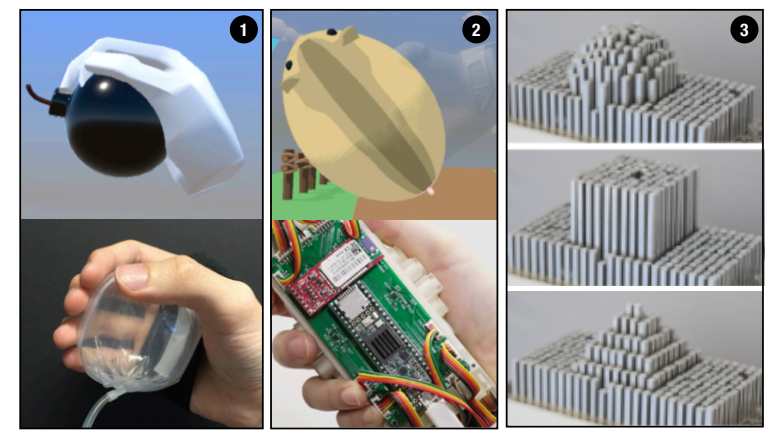

Figure 3: Simulating Objects. (1) A controller with an inflatable prop in the user's palm simulates holding a bomb [153]. (2) A pin-based interface shaped as a ball interacts in the user palm to replicate a hamster [185]. (3) Different primitives (ball, cube, pyramid) are displayed on a 2.5D tabletop [138]. and constrain their respective positions to each other [144, 171]. Contactless technology has also been developed for simulating shapes. While studies demonstrated that interacting with barehands increased the user's cognitive load [52], combining barehands interactions with haptic feedback actually enhances the users involvement. Since haptic feedback does require contact, "contactless" technology defines an interaction where the users are unencumbered, as per Krueger's postulate [172], and ultrasounds are sent to their hands, for them to perceive shapes on their skin, without a physical prop contact [117] (Figure 2 - 4).

These unencumbered methods are also achieved through shapechanging interfaces, for instance with balloons arrays [151] or 2.5D tabletops (Figure 3 - 3, Figure 1 - 5) [48, 76, 138]. These latter are constituted from pins, that raise and lower themselves to replicate different shapes. In the same regard, swarm interfaces rearrange themselves to display different shapes. These have mainly been developed in the real world $[43,79,86,99,149,150]$ but slowly take off as VR user interfaces [190] (Figure 1 - 4). Indeed, while these latter devices are used as desktop interfaces, the swarm robot idea has extended to the air, with drones for instance [54, 70, 81, 122, 160]. All of these previous interfaces embrace the Roboxel principle enunciated in Robotic Graphics [101]: "cellular robots that dynamically configure themselves into the desired shape and size".

6.1.3 Object Primitives, (Figure 2 - 5). Finally, a user can interact with object primitives. These represent the simplest geometries available: circle, cube, pyramid, cylinder, torus. Simply feeling an orientation through the fingertips provides the required information to understand an object shape, in an exploration task for instance. Panels with diverse orientations can hence be displayed for a user to explore various objects in a virtual environment [30] (Figure 2 - 5) or directly encounter the user at their position of interest $[183,184]$.

On the opposite, a bare-hands manipulation task requires multiple primitives to be available at the same time within the hand vicinity. This is why the exploitation of real objects is necessary.

\subsection{Exploiting Real Objects}

Passive haptics [73], ie the use of passive props, consist in placing real objects corresponding to their exact virtual match at their virtual position. Insko demonstrated that passive haptics enhanced 
the virtual environment [73]. Nonetheless, this does suffer from a main limitation: substituting the physical environment for a virtual one [135] requires a thorough mapping of objects shapes, sizes, textures, and requires numerous props [110]. This can be done with real objects in simulation rooms for instance (e.g plane cockpit, motorcycle), but cheaper methods need to be implemented to facilitate their use in other fields.

6.2.1 Object Primitives, (Figure 2 - 6). One solution is to extract the primitives of the objects that are already available in the physical environment, to map virtual objects of the approximate same primitive over them [69] (Figure 2 - 6).

6.2.2 Visuo-Proprioceptive Illusions \& Pseudo Haptics. The number of props within the environment can also be reduced, while letting the users interact at different positions of the physical world. It is possible to leverage the vision over haptics and modify the users' proprioception to redirect their trajectory [13, 56, 61, 82-84]. A user might perceive multiple distinct cubes for instance, while interacting with a single one. On the same principle, the user hand displacement can be redirected at an angle, up-/down-scaled [4, $21]$, or slowed down for friction or weight perception [113, 125]. These techniques also allow for the exploration and manipulation of various shapes: models can for instance be added to enable complex virtual shapes to be mapped over real physical objects boundaries [189]. The user can also be redirected to pinch a multi-primitive object (cubic, pyramidal and cylindrical) from different locations, which theoretically widens the variety of available props with a single one [40]. On the same principle, pseudo-haptics allow to modify the users' shape $[15,16]$ or texture [41] perceptions when interacting with a physical prop.

6.2.3 Displacing Objects, (Figure 2 - 7). Whenever objects are indeed available within the environment, various directions are available to displace them. This displacement allows for mapping one physical object over multiple ones, but also to display a multitude of props. These directions embrace the Robotic Shape Display principle from Robotic Graphics [101]: "a robot that can reach any location of a virtual desktop with an end-effector" and matches the user's object of interest.

Their usability have been validated through a Wizard-of-Oz implementation, where human operators move real objects or even people around a Room-scale VR arena to encounter the users [31] (Figure 4 - 2). The users themselves can also reconfigure and actuate real props [28].

Robotic Shape Displays, RSDs, are also called encountered-type of haptic devices, as they literally encounter the users at their object of interest to provide haptic feedback. They allow to display real pieces of material $[5,11]$, physical props to simulate walls $[24,80,178]$, or even display furniture [148] or untethered objects $[24,64,65,72]$, that can be interacted with each other.

\section{CONCEPTION COST}

In practice, designers have to trade-off their interaction design space with implementation and operational costs in the conception phase. Implementation costs include technical aspects related to the acceptability of an haptic solution such as safety, robustness and ease-of-use [42]. For instance, actuated haptic solutions require a special attention regarding this criterion. Operation costs include the financial and human cost for using a haptic solution. The financial cost is measured through the cost of the haptic device and additional elements such as motion capture systems to precisely track the users' hand or the prior preparation of required props. Human cost refers to both labour time and number of human operators required during the user's interactions. For instance, actuated haptic solutions generally do not require human operators (low human cost) but might be mechanically expensive.

In this section, we use our two-dimension design space (Table 1) to discuss haptic solutions according to their conception cost. As non-actuated solutions globally share the same approaches and have a low implementation cost, we discuss them together in the "No Robotics" subsection.

\subsection{No Robotics}

Regarding implementation costs, all non-actuated haptic solutions are safe, robust and easy-to-use. We depict here an important design choice when opting for these solutions: either the designer relies on graphics solutions, leveraging vision cues over haptic ones, or needs operators to displace or change the interactable props (see Table 1).

7.1.1 Passive Props. Passive props [73] only consist in placing real objects corresponding to their exact virtual match at their virtual position. They provide a natural way of interacting through the objects' natural affordances [107]. They however are limited to the available objects within the scene as they are not actuated. They only can be used in a scenario-based experience, where the target is known in advance. The environment hence requires a prop for each available virtual object.

7.1.2 Shape Simulation, Pseudo-Haptics, Visuo-Haptic Illusions, Object Primitives. For graphics solutions, users are redirected towards their object of interest [13] using visuo-haptic illusions. However, physically overlaying a prop or primitive over a virtual object has a tracking cost, which usually relies on trackers which can be operationally costly (eg Optitrack [108] or HTC Trackers).

Otherwise, the users intentions have to be predicted for the interaction to occur. The users hands are then redirected to the appropriate motionless prop, for them to explore their object of interest [30]. Operationally, the cost only relies on the proxy fabrication (Figure 2 - 5). These implementations offer various scenarios in terms of interaction (even non-deterministic), at an affordable cost.

7.1.3 Surface Haptic Displays. These techniques exclusively allow for exploration through multiple haptic features such as friction or textures. They also can integrate a tablet or a smartphone [128], on which the user can interact at any location.

7.1.4 Human Actuators. This technique consists in using human operators to displace props in the VR arena. The designers however come across reliability and speed issues with these operators. Even though they only are used in scenario-based experiences, delay mechanisms based on graphics need to be implemented [31] (Figure 4 - 2) to overcome these issues. Conceptually, they broaden the interaction scope, however this solution is operationally very costly. 


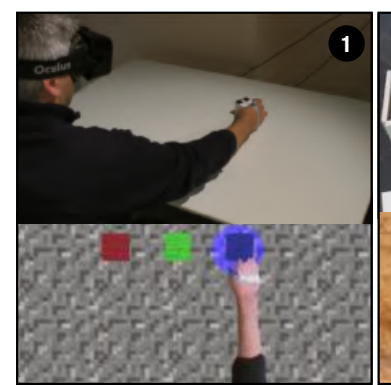

No actuation

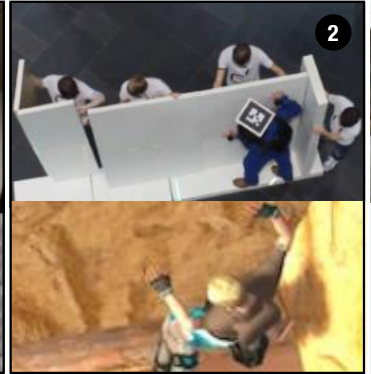

WoZ/Humans

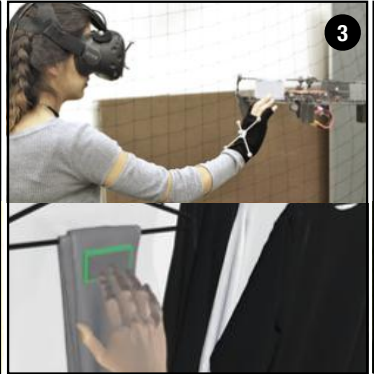

Drone

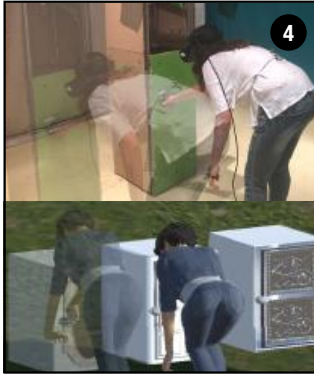

XY Robot

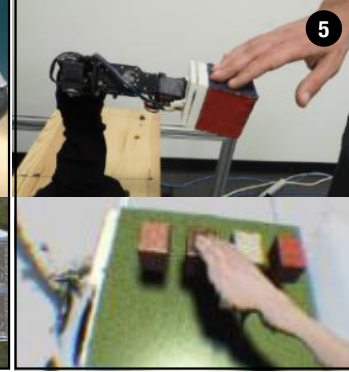

Robotic Arm

Figure 4: Degree of Actuation. (1) No actuation is available. The user's hand is redirected to touch a passive prop that cannot move [13]. The implementation of this technique relies exclusively on a software development leveraging the vision cues; (2) Human actuators are used to illustrate the Robotic Graphics [101] principle with a Wizard of Oz technique [31]. They carry props for the user to feel a real continuous wall; Encountered-type of haptic devices (3-5): (3) A drone encounters the users' hand for exploring passive props; (4) A cartesian robot displaces itself autonomously for users to interact with physical props [24]; (5) A robotic arm with multiple degrees of freedom displaces itself to encounter the users' hand, and rotates its shape-approximation device to provide the right material [11].

7.1.5 Real Props Reassignment. Instead of using a tracking system for passive props, a depth camera for instance allows to reassign props to different virtual objects of the same primitive [69] (Figure 2 - 6). The objects are hence all available to be interacted with. This drastically reduces the operational costs as they only rely on computer vision. This enables non-deterministic scenarios as the real world is literally substituted for a virtual one [135] and objects can be reassigned with virtual:physical [65] mappings.

\subsection{Robotics \& No Real Objects}

This section gathers technologies simulating the virtual environment through actuation: they replicate it to constrain the users.

7.2.1 Desktop Haptic Interfaces. The SPIDAR [127], the Virtuose [62] and other classic desktop haptic interfaces are already compared in multiple surveys $[42,132,168]$ (see Figure 2 - 1). They are safe as they are controlled by the user and only constrain their arm movements with kinesthetic feedback and adapt to any available object from the virtual scene (non-deterministic scenarios). They show a high perceived stiffness and robustness, but remain really expensive $(>10 \mathrm{k} \$)$.

7.2.2 Shape-Changing Interfaces, Roboxels, 2.5D Tabletops. These technologies present a high perceived stiffness and change their shapes accordingly with the virtual environment [47, 91]. They hence do not require any operator and allow for non-deterministic scenarios whenever their displacements are enabled [138] (see Figure 3 - 3). They are however complex to build and require multiple motors as they are composed of arrays of numerous pins, which define their haptic fidelity resolution. Even though they present high voltages, they remain safe around the users. As they require bare-hands interactions, they hence show a high ease of use.

7.2.3 Wearables, Controllers, EMS.. These rely on small torques, which are sufficient to constrain the users body parts. They are safe and easy to use, but in return are not robust enough to resist to users' actions. As they are continuously changing the users' haptic perception, they do allow non-deterministic scenarios and change their rendered stiffness and rigidity as a function of the distance to a virtual prop [39, 85]. A customised controller usually relies on 3D printed parts and small servomotors and can be easily replicated [146] (Figure 2 - 2,3; Figure 3 - 1,2).

7.2.4 Mid-Air Haptics. Providing contactless interactions, mid-air haptics also provide a high level of safety around the user. They however do not allow to navigate the VR environment, and hence cannot consider non-deterministic scenarios. Their robustness is very low, as they send ultrasounds to the users and do not physically constrain them [117].

7.2.5 Inflatable Floor. The floor topology can be modified and inflated to create interactions at the body-scale [154]. The users cannot inflate them, however they can push some tiles down and hence, edit them. These are safe, though they do not provide a wide range of interactions, but offer multiple static body postures.

\subsection{Robotics \& Real Objects}

In this subsection, we detail the different types of Robotic Shapes Displays - otherwise known as "encountered-type of haptic devices", mentioned in the Table 1 . First, these interfaces move to encounter the users: this feature optimises their ease of use. Second, as these interfaces move within the user vicinity, safety concerns are raised in this section, depending on the interfaces robustness. Encountered-type of haptic devices combine different types of interaction techniques: they can provide the users with passive props, textures or primitives, and allow navigation, exploration, manipulation tasks. Their mechanical implementations offer a good repeatability and reliability.

7.3.1 Cartesian Robot: In [24], CoVR, a physical column mounted over a Cartesian XY ceiling robot enables interactions at any height and any position of a room-scale VR arena (see Figure 1 - 2; Figure 4 - 4). This implementation presents a high perceived stiffness, and because it carries passive props around the arena, enables a 
high fidelity haptic rendering. It displays high accuracy and speed, and presents an algorithm which optimises the column's displacements as a function of the users intentions. It hence enables nondeterministic scenarios. Safety measures have been validated in the field. In practice, the column's celerity is decreasing around the user, as it is repulsed by this latter. Its software implementation ensures a safe environment for the user to perambulate in the arena without unexpected collision. However, in order to display many props in different scenarios, an operator is required to create panels and modify them. The materials however remain cheap, and even though its structure and motors are more expensive than 3D printed cases and servomotors, as per customised controllers for instance, this solution provides a wide range of interactions.

7.3.2 Robotic Arm: A robotic arm provides more degrees of freedom than the previous Cartesian robot. This primarily means a higher cost and a higher safety risk. For instance, H-Wall, using a Kuka LBR Iiwa robot, presents high motor torques and can hence increase the safety risks around the users. This implementation hence does not allow non-deterministic scenarios, and presents either a wall or a revolving door to the user, with a high robustness. Implementations with smaller torques, such as $[11,166]$ are safer but display a reduced perceived stiffness. The use-cases for all these interactions are hence drastically different: $\mathrm{H}$-Wall simulates a rigid wall while VRRobot [166] and Snake Charmer [11] (Figure 4 - 5) present more interaction opportunities. This latter is also the single Robotic Shape Display that autonomously changes its end-effector, without an operator.

7.3.3 Drones, Swarm Robots, Mobile Platforms: With drones, the interactions are limited to the available props, for instance with a single wall at a given position [178]. Going from an active mode (flying) to a passive one (graspable by the user) has a long delay (10s) [5], which on top of the safety concerns, does not allow nondeterministic scenarios. [159] however allows the user to change the drone trajectory to fetch and magnetically recover an object of interest. Their accuracy and speed are limited [54, 122] compared to the previous grounded interfaces, and can require dynamic redirection techniques to improve their performances [5]. As they are ungrounded, they do not have a high robustness nor perceived stiffness. This is also valid for mobile robots, such as [55, 65], which only display passive props. To decrease the conception cost, existing vacuuming robots are used as mobile platforms in [170, 181]. Designers can choose to duplicate them, as swarm robots, to enable non-deterministic scenarios [148]. These are safe to use around the users, as their speed and robustness are limited. Instead of swarm mobile interfaces, a merry-go-round platform can also be designed to display various props at an equidistant position from the user [72]. All of the previous interfaces require an operator cost on top of their mechanical and software ones, to modify the interactable props available, depending on the use-cases.

On the opposite, [190] proposes autonomous reconfigurable interfaces intertwining both Robotic Shape Displays and Roboxels [101] principles to get rid of the operator cost (see Figure 1 - 4). These small robotic volume elements reconfigure themselves into the users objects of interest. They have a sufficient perceived stiffness to represent objects, but are not robust enough to resist to body-scaled forces, for instance to simulate a rigid wall.

\section{EVALUATION PROTOCOLS}

On top of choosing from the different trade-offs between conception and interaction opportunities, the designer also needs to pick-up an evaluation protocol. These protocols depend on the VR usecases. For instance, the haptic benefits for medical or industrial assembly training can be evaluated against a real experience condition [112], with criteria such as completion time, number of errors, user cognitive load [59]. On the opposite, the haptic benefits for a gaming experience are more likely to be evaluated through immersion and presence, comparing "with/without haptics" conditions [31]. Although some papers do compare multiple haptic displays $[44,161]$, we point out the lack of referenced evaluation protocols for evaluating haptic solutions in VR.

\subsection{Current Reference Evaluation Methods}

The most common evaluation methods in VR are the SUS or WS presence questionnaires $[140,175]$. These questionnaires mainly focus on graphics rendering and only two Likert-scale questions actually focus on haptic feedback: "How well could you actively survey the VE using touch?" and "How well could you manipulate objects in the VE?". Besides, most of the above technologies are evaluated against "no haptic feedback", hence the results can seem biased and most of all, expected. This justifies why some implementations provide results on single parts of the questionnaire, or arbitrarily combine their results [34] with new subsections (eg "ability to examine/act") or tasks specific questions (eg "How realistic was it to feel different textures?).

\subsection{Evaluation Recommendations}

Haptics should be more incorporated into the different factors enunciated in [175] ("Control, Sensory, Distraction, Realism"). In this direction, Kim et al. defined the Haptic Experience model [78], where they take into account both of the designer and user experiences. It depicts how Design parameters ("timeliness, intensity, density and timbre") impact Usability requirements ("utility, causality, consistency, saliency") and target Experiential dimensions ("harmony, expressivity, autotelics, immersion, realism") on the user's side.

In the same regards, we propose additional guidelines to evaluate haptic solutions in VR experiments (see Table 2). We believe that the different elements of interaction opportunities should be added to the users control parameters.

In the sensory factors, the number of haptic features available should be added (eg shape, texture, friction, temperature), in line with their quality, in terms of "timeliness, intensity, density and timbre". The usability requirements should identify the use-cases and number of scenarios with the proposed solutions. Hence, a good evaluation of the interface timeliness and usability should anticipate future deployments and avoid unnecessary developments.

\section{EXAMPLES: ENCOUNTERED-TYPE OF HAPTIC DEVICES}

We propose in this section to compare four encountered-type of haptic devices: Beyond the Force (BTF) drone [5] (Figure 4 - 3), ShapeShift [138] (Figure 3 - 3), Snake Charmer [11] (Figure 4 - 5), and CoVR [24] (Figure 4 - 4). 


\begin{tabular}{|c|c|c|c|c|c|c|c|c|c|c|c|c|c|c|c|c|}
\hline & $\begin{array}{l}\text { Navigation } \\
\text { Workspace }\end{array}$ & $\begin{array}{l}\text { Haptic } \\
\text { Features }\end{array}$ & Exploration & Manipula & Edition & $\begin{array}{l}\text { Whole.BO } \\
\text { involvem }\end{array}$ & $\begin{array}{l}\text { Non- } \\
\text { Determir } \\
\text { Scenarit }\end{array}$ & $\begin{array}{l}\text { Number of } \\
\text { Props }\end{array}$ & $\begin{array}{l}\text { passive } \\
\text { Haptics }\end{array}$ & operator & $\begin{array}{l}\text { Deployment } \\
\text { use-cases }\end{array}$ & Robustness & $\begin{array}{l}\text { Accuracy } \\
\text { speed }\end{array}$ & sarety & Ease-of-Use & $\begin{array}{l}\text { Evaluation } \\
\text { Method }\end{array}$ \\
\hline $\begin{array}{l}\text { Beyond } \\
\text { the Force }\end{array}$ & $2 \mathrm{~m}^{3}$ & ++ & ++ & + & - & - & - & $\begin{array}{c}\text { Prepared } \\
\text { prior to use }\end{array}$ & Yes & Yes & ' & - & - & - & +++ & \begin{tabular}{|l} 
Noise \& Fear \\
Acceptance
\end{tabular} \\
\hline \multirow{2}{*}{$\begin{array}{c}\text { ShapeShift } \\
\text { Snake } \\
\text { Charmer }\end{array}$} & Desktop & + & +++ & + & + & - & ++ & $\infty$ & No & No & \begin{tabular}{c|} 
3D Terrain \\
VolumetricData
\end{tabular} & ++ & + & +++ & +++ & $\begin{array}{c}\text { Shape } \\
\text { Recognition }\end{array}$ \\
\hline & Desktop & +++ & +++ & ++ & - & - & ++ & $\begin{array}{c}\text { Prepared } \\
\text { prior to use }\end{array}$ & Yes & No & Training & + & + & ++ & +++ & $\begin{array}{l}\text { No Formal } \\
\text { Evaluation }\end{array}$ \\
\hline CoVR & $30 \mathrm{~m}^{3}$ & +++ & +++ & +++ & - & +++ & +++ & $\begin{array}{c}\text { Prepared } \\
\text { prior to use }\end{array}$ & Yes & Yes & $\begin{array}{l}\text { Arcade } \\
\text { Training }\end{array}$ & +++ & ++ & + & +++ & $\begin{array}{c}\text { Users } \\
\text { Enjoyment }\end{array}$ \\
\hline
\end{tabular}

Table 2: Comparison \& Evaluation of 4 Encountered-type of Haptic Devices, according to the "Evaluation section" parameters.

In terms of interactions and number of props, the drone is the most limited one. Indeed, because of both safety and implementation limitations, it only enables free navigation in a reduced workspace. It also allows exploration (through textures) and manipulation tasks. However, the manipulation task is at the moment limited to a single light object as BTF cannot handle large embedded masses yet. Whenever grabbed, it does not provide a haptic transparency [63] during the interactions because of its thrust and inertia. For the users to perform different tasks, an operator needs to manually change the drone configuration. Its mechanical implementation does not provide a sufficient speed for overlaying virtual props in non-deterministic scenarios, but its accuracy is also unsatisfactory and requires dynamic redirection techniques for the interactions to occur. It also provides unwanted noise and wind, which reduces the interaction realism.

ShapeShift [138] is drastically different: it is a 2.5D desktop interface that displaces itself. Even though a drone is theoretically available in an infinite workspace, in practice they do share approximately the same one. As [138] relies on a shape-changing interface, no operator is required and it shape changes itself to overlay the users' virtual objects of interest, in non-deterministic scenarios. It allows a free navigation at a desktop scale, as well as bimanual manipulation and exploration. Both of these devices haptic transparency are limited as they are ungrounded solutions. We believe that ShapeShift could be updated to allow Edition tasks, by synchronising the users force actions with the actuated pins stiffness. In terms of haptic features, it simulates shapes and stimulates both tactile and kinesthetic cues. As per all 2.5D tabletops, it can be used in various applications: 3D terrain exploration, volumetric data etc. Its resolution seems promising as its studies shows successful object recognition and haptic search. The same interactions are available at a desktop scale with Snake Charmer [11], which provides a wide range of props and stimulation, as each of its end-effector include 6 faces with various interaction opportunities (textures to explore, buttons to push, heater and fan to perceive temperature, handle and lightbulb to grasp and manipulate...). It also can change its shape approximation device, SAD (ie its end-effector), autonomously, using magnets. It follows the user hand and orient the expected interaction face of its $S A D$ prior to the interactions: it hence enables non-deterministic scenarios. Besides, Snake Charmer has a promising future regarding its deployment: LobbyBot [1], is already in the Renault industry research lab, to enable VR haptic feedback in the automotive industry.

Finally, CoVR [24] enables the largest workspace as well as the highest range of interactions. The user is free to navigate in a
$30 \mathrm{~m}^{3} \mathrm{VR}$ arena, and CoVR predicts and physically overlays his object of interest prior to interaction. These interactions include tactile exploration, manipulation of untethered objects (full haptic transparency), body postures. Indeed, CoVR is robust enough to resist body-scaled users, and shows over a $100 \mathrm{~N}$ perceived stiffness and can carry over $80 \mathrm{~kg}$ of embedded mass. CoVR can also initiate the interactions with the users, and is strong enough to lead the users through forces or even to transport them. Moreover, with the appropriate physical:virtual mapping [65], one physical prop can overlay multiple virtual ones of the same approximate primitive without redirection techniques. It however requires an operator to create, assemble and display panels on its sides.

Room-scale VR becomes more and more relevant, and Snake Charmer could benefit from being attached to an interface such as CoVR. Similarly, intertwining CoVR with a robotic arm autonomously changing its SAD like Snake Charmer or with a shape-changing interface could reduce its operational costs. This would display all of the Robotics Graphics concept capabilities.

\section{CONCLUSION}

We analysed in this paper haptic interactions in VR and their corresponding haptic solutions. We analyzed them from both the user and designer perspectives by considering interaction opportunities and visuo-haptic consistency, as well as implementation and operation costs. We proposed a novel framework to classify haptic displays, through a two-dimension design space: the interfaces' degree of physicality and degree of actuation.

We then evaluated these latter solutions from an interaction and conception perspectives. Implementation-wise, we evaluated the interfaces robustness, their ease of use as well as their safety considerations. From an operation perspective, we also evaluated the costs of the proposed solutions.

This survey highlights the variety of props, tasks and haptic features that a haptic solution can potentially provide in VR. This survey can be used to analytically evaluate the existing haptic interactions. It can also help VR designers to choose the desired haptic interaction and/or haptic solution depending on their needs (tasks, workspace, use-cases etc).

We believe that combining multiple haptic solutions benefits the user experience, as it optimises the above criteria. Encountered-type of haptic interfaces were then highlighted as they already combine multiple interaction techniques: they displace passive props in potentially large VR arenas and allow for numerous tasks, such as navigation, exploration, manipulation, and even allow the user to be interacted with. 


\section{REFERENCES}

[1] [n.d.]. renault. https://www.clarte-lab.fr/component/tags/tag/renault

[2] 2019. CyberGrasp. http://www.cyberglovesystems.com/cybergrasp

[3] 2019. Teslasuit | Full body haptic VR suit for motion capture and training https://teslasuit.io/

[4] Parastoo Abtahi and Sean Follmer. 2018. Visuo-Haptic Illusions for Improving the Perceived Performance of Shape Displays. In Proceedings of the $2018 \mathrm{CH}$ Conference on Human Factors in Computing Systems - CHI '18. ACM Press, Montreal QC, Canada, 1-13. https://doi.org/10.1145/3173574.3173724

[5] Parastoo Abtahi, Benoit Landry, Jackie (Junrui) Yang, Marco Pavone, Sean Follmer, and James A. Landay. 2019. Beyond The Force: Using Quadcopters to Appropriate Objects and the Environment for Haptics in Virtual Reality. In Proceedings of the 2019 CHI Conference on Human Factors in Computing Systems - CHI '19. ACM Press, Glasgow, Scotland Uk, 1-13. https://doi.org/10.1145/ 3290605.3300589

[6] Merwan Achibet, Adrien Girard, Anthony Talvas, Maud Marchal, and Anatole Lecuyer. 2015. Elastic-Arm: Human-scale passive haptic feedback for augmenting interaction and perception in virtual environments. In 2015 IEEE Virtual Reality (VR). IEEE, Arles, Camargue, Provence, France, 63-68. https: //doi.org/10.1109/VR.2015.7223325

[7] Merwan Achibet, Benoit Le Gouis, Maud Marchal, Pierre-Alexandre Leziart, Ferran Argelaguet, Adrien Girard, Anatole Lecuyer, and Hiroyuki Kajimoto. 2017. FlexiFingers: Multi-finger interaction in VR combining passive haptics and pseudo-haptics. In 2017 IEEE Symposium on 3D User Interfaces (3DUI). IEEE, Los Angeles, CA, USA, 103-106. https://doi.org/10.1109/3DUI.2017.7893325

[8] Merwan Achibet, Maud Marchal, Ferran Argelaguet, and Anatole Lecuyer. 2014 The Virtual Mitten: A novel interaction paradigm for visuo-haptic manipulation of objects using grip force. In 2014 IEEE Symposium on 3D User Interfaces (3DUI). IEEE, MN, USA, 59-66. https://doi.org/10.1109/3DUI.2014.6798843

[9] Dmitry Alexandrovsky, Susanne Putze, Michael Bonfert, Sebastian Höffner, Pitt Michelmann, Dirk Wenig, Rainer Malaka, and Jan David Smeddinck. 2020. Examining Design Choices of Questionnaires in VR User Studies. In Proceedings of the 2020 CHI Conference on Human Factors in Computing Systems. ACM, Honolulu HI USA, 1-21. https://doi.org/10.1145/3313831.3376260

[10] E. Amirpour, M. Savabi, A. Saboukhi, M. Rahimi Gorii, H. Ghafarirad, R. Fesharakifard, and S. Mehdi Rezaei. 2019. Design and Optimization of a Multi-DOF Hand Exoskeleton for Haptic Applications. In 2019 7th International Confer ence on Robotics and Mechatronics (ICRoM). 270-275. https://doi.org/10.1109/ ICRoM48714.2019.9071884 ISSN: 2572-6889.

[11] Bruno Araujo, Ricardo Jota, Varun Perumal, Jia Xian Yao, Karan Singh, and Daniel Wigdor. 2016. Snake Charmer: Physically Enabling Virtual Objects. In Proceedings of the TEI '16: Tenth International Conference on Tangible, Embedded, and Embodied Interaction - TEI'16. ACM Press, Eindhoven, Netherlands, 218-226. https://doi.org/10.1145/2839462.2839484

[12] Jonas Auda, Max Pascher, and Stefan Schneegass. 2019. Around the (Virtual) World: Infinite Walking in Virtual Reality Using Electrical Muscle Stimulation. In Proceedings of the 2019 CHI Conference on Human Factors in Computing Systems - CHI '19. ACM Press, Glasgow, Scotland Uk, 1-8. https://doi.org/10.1145/ 3290605.3300661

[13] Mahdi Azmandian, Mark Hancock, Hrvoje Benko, Eyal Ofek, and Andrew D. Wilson. 2016. Haptic Retargeting: Dynamic Repurposing of Passive Haptics for Enhanced Virtual Reality Experiences. In Proceedings of the 2016 CHI Conference on Human Factors in Computing Systems - CHI '16. ACM Press, Santa Clara, California, USA, 1968-1979. https://doi.org/10.1145/2858036.2858226

[14] Marc Baloup, Veïs Oudjail, Thomas Pietrzak, and Géry Casiez. 2018. Pointing techniques for distant targets in virtual reality. In Proceedings of the 30th Conference on l'Interaction Homme-Machine - IHM '18. ACM Press, Brest, France, 100-107. https://doi.org/10.1145/3286689.3286696

[15] Y. Ban, T. Kajinami, T. Narumi, T. Tanikawa, and M. Hirose. 2012. Modifying an identified curved surface shape using pseudo-haptic effect. In 2012 IEEE Haptics Symposium (HAPTICS). 211-216. https://doi.org/10.1109/HAPTIC.2012.6183793

[16] Yuki Ban, Takuji Narumi, Tomohiro Tanikawa, and Michitaka Hirose. 2012. Modifying an identified position of edged shapes using pseudo-haptic effects. In Proceedings of the 18th ACM symposium on Virtual reality software and technology - VRST '12. ACM Press, Toronto, Ontario, Canada, 93. https://doi.org/10.1145/ 2407336.2407353

[17] Gareth Barnaby and Anne Roudaut. 2019. Mantis: A Scalable, Lightweight and Accessible Architecture to Build Multiform Force Feedback Systems. In Proceedings of the 32nd Annual ACM Symposium on User Interface Software and Technology - UIST '19. ACM Press, New Orleans, LA, USA, 937-948. https: //doi.org/10.1145/3332165.3347909

[18] Olivier Bau, Ivan Poupyrev, Ali Israr, and Chris Harrison. 2010. TeslaTouch: electrovibration for touch surfaces. In Proceedings of the 23nd annual ACM symposium on User interface software and technology - UIST '10. ACM Press, New York, New York, USA, 283. https://doi.org/10.1145/1866029.1866074
[19] Hrvoje Benko, Christian Holz, Mike Sinclair, and Eyal Ofek. 2016. NormalTouch and TextureTouch: High-fidelity 3D Haptic Shape Rendering on Handheld Virtual Reality Controllers. In Proceedings of the 29th Annual Symposium on User Interface Software and Technology - UIST '16. ACM Press, Tokyo, Japan, 717-728. https://doi.org/10.1145/2984511.2984526

[20] Leif P. Berg and Judy M. Vance. 2017. Industry use of virtual reality in product design and manufacturing: a survey. Virtual Reality 21, 1 (March 2017), 1-17. https://doi.org/10.1007/s10055-016-0293-9

[21] Joanna Bergström, Aske Mottelson, and Jarrod Knibbe. 2019. Resized Grasping in VR: Estimating Thresholds for Object Discrimination. In Proceedings of the 32nd Annual ACM Symposium on User Interface Software and Technology. ACM, New Orleans LA USA, 1175-1183. https://doi.org/10.1145/3332165.3347939

[22] A. Bloomfield, Yu Deng, J. Wampler, P. Rondot, D. Harth, M. McManus, and N. Badler. 2003. A taxonomy and comparison of haptic actions for disassembly tasks. In IEEE Virtual Reality, 2003. Proceedings. IEEE Comput. Soc, Los Angeles, CA, USA, 225-231. https://doi.org/10.1109/VR.2003.1191143

[23] Mette Boldt, Boxuan Liu, Tram Nguyen, Alina Panova, Ramneek Singh, Alexander Steenbergen, Rainer Malaka, Jan Smeddinck, Michael Bonfert, Inga Lehne, Melina Cahnbley, Kim Korschinq, Loannis Bikas, Stefan Finke, Martin Hanci, and Valentin Kraft. 2018. You Shall Not Pass: Non-Intrusive Feedback for Virtual Walls in VR Environments with Room-Scale Mapping. In 2018 IEEE Conference on Virtual Reality and 3D User Interfaces (VR). IEEE, Reutlingen, 143-150. https://doi.org/10.1109/VR.2018.8446177

[24] Elodie Bouzbib, Gilles Bailly, Sinan Haliyo, and Pascal Frey. 2020. CoVR: A Large-Scale Force-Feedback Robotic Interface for Non-Deterministic Scenarios in VR. In Proceedings of the 33rd Annual ACM Symposium on User Interface Software and Technology. ACM, Virtual Event USA, 209-222. https://doi.org/10. $1145 / 3379337.3415891$

[25] D.A. Bowman and C.A. Wingrave. 2001. Design and evaluation of menu systems for immersive virtual environments. In Proceedings IEEE Virtual Reality 2001. IEEE Comput. Soc, Yokohama, Japan, 149-156. https://doi.org/10.1109/VR.2001. 913781

[26] Steve Bryson. 2005. Direct Manipulation in Virtual Reality. In Visualization Handbook. Elsevier, 413-430. https://doi.org/10.1016/B978-012387582-2/50023$\mathrm{X}$

[27] Lung-Pan Cheng. 2019. VRoamer: Generating On-The-Fly VR Experiences While Walking inside Large, Unknown Real-World Building Environments. (2019), 8.

[28] Lung-Pan Cheng, Li Chang, Sebastian Marwecki, and Patrick Baudisch. 2018. iTurk: Turning Passive Haptics into Active Haptics by Making Users Reconfigure Props in Virtual Reality. In Proceedings of the 2018 CHI Conference on Human Factors in Computing Systems - CHI '18. ACM Press, Montreal QC, Canada, 1-10. https://doi.org/10.1145/3173574.3173663

[29] Lung-Pan Cheng, Patrick Lühne, Pedro Lopes, Christoph Sterz, and Patrick Baudisch. 2014. Haptic Turk: a Motion Platform Based on People. (2014), 11.

[30] Lung-Pan Cheng, Eyal Ofek, Christian Holz, Hrvoje Benko, and Andrew D. Wilson. 2017. Sparse Haptic Proxy: Touch Feedback in Virtual Environments Using a General Passive Prop. In Proceedings of the 2017 CHI Conference on Human Factors in Computing Systems - CHI '17. ACM Press, Denver, Colorado, USA, 3718-3728. https://doi.org/10.1145/3025453.3025753

[31] Lung-Pan Cheng, Thijs Roumen, Hannes Rantzsch, Sven Köhler, Patrick Schmidt, Robert Kovacs, Johannes Jasper, Jonas Kemper, and Patrick Baudisch. 2015. TurkDeck: Physical Virtual Reality Based on People. In Proceedings of the 28th Annual ACM Symposium on User Interface Software \& Technology - UIST '15. ACM Press, Daegu, Kyungpook, Republic of Korea, 417-426. https://doi.org/10. $1145 / 2807442.2807463$

[32] Inrak Choi, Heather Culbertson, Mark R. Miller, Alex Olwal, and Sean Follmer. 2017. Grabity: A Wearable Haptic Interface for Simulating Weight and Grasping in Virtual Reality. In Proceedings of the 30th Annual ACM Symposium on User Interface Software and Technology - UIST '17. ACM Press, Qu\&\#233;bec City, QC, Canada, 119-130. https://doi.org/10.1145/3126594.3126599

[33] Inrak Choi, Elliot W. Hawkes, David L. Christensen, Christopher J. Ploch, and Sean Follmer. 2016. Wolverine: A wearable haptic interface for grasping in virtual reality. In 2016 IEEE/RSF International Conference on Intelligent Robots and Systems (IROS). IEEE, Daejeon, South Korea, 986-993. https://doi.org/10. 1109/IROS.2016.7759169

[34] Inrak Choi, Eyal Ofek, Hrvoje Benko, Mike Sinclair, and Christian Holz. 2018. CLAW: A Multifunctional Handheld Haptic Controller for Grasping, Touching, and Triggering in Virtual Reality. In Proceedings of the $2018 \mathrm{CHI}$ Conference on Human Factors in Computing Systems - CHI '18. ACM Press, Montreal QC, Canada, 1-13. https://doi.org/10.1145/3173574.3174228

[35] Timothy R. Coles, Dwight Meglan, and Nigel W. John. 2011. The Role of Haptics in Medical Training Simulators: A Survey of the State of the Art. IEEE Transactions on Haptics 4, 1 (Jan. 2011), 51-66. https://doi.org/10.1109/TOH. 2010.19

[36] Fabien Danieau, Julien Fleureau, Philippe Guillotel, Nicolas Mollet, Anatole Lécuyer, and Marc Christie. 2012. HapSeat: producing motion sensation with multiple force-feedback devices embedded in a seat. In Proceedings of the 18th 
ACM symposium on Virtual reality software and technology - VRST '12. ACM Press, Toronto, Ontario, Canada, 69. https://doi.org/10.1145/2407336.2407350

[37] Fabien Danieau, Philippe Guillotel, Olivier Dumas, Thomas Lopez, Bertrand Leroy, and Nicolas Mollet. 2018. HFX studio: haptic editor for full-body immersive experiences. In Proceedings of the 24th ACM Symposium on Virtual Reality Software and Technology - VRST '18. ACM Press, Tokyo, Japan, 1-9. https://doi.org/10.1145/3281505.3281518

[38] Bruno R. De Araújo, Géry Casiez, Joaquim A. Jorge, and Martin Hachet. 2013 Mockup Builder: 3D modeling on and above the surface. Computers \& Graphics 37, 3 (May 2013), 165-178. https://doi.org/10.1016/j.cag.2012.12.005

[39] Xavier de Tinguy, Thomas Howard, Claudio Pacchierotti, Maud Marchal, and Anatole Lécuyer. 2020. WeATaViX: WEarable Actuated TAngibles for VIrtual reality eXperiences. (2020), 9.

[40] Xavier de Tinguy, Claudio Pacchierotti, Maud Marchal, and Anatole Lecuyer 2019. Toward Universal Tangible Objects: Optimizing Haptic Pinching Sensations in 3D Interaction. In 2019 IEEE Conference on Virtual Reality and 3D User Interfaces (VR). IEEE, Osaka, Japan, 321-330. https://doi.org/10.1109/VR.2019. 8798205

[41] Donald Degraen, André Zenner, and Antonio Krüger. 2019. Enhancing Texture Perception in Virtual Reality Using 3D-Printed Hair Structures. In Proceedings of the 2019 CHI Conference on Human Factors in Computing Systems - CHI '19. ACM Press, Glasgow, Scotland Uk, 1-12. https://doi.org/10.1145/3290605.3300479

[42] Lionel Dominjon, Jérôme Perret, and Anatole Lécuyer. 2007. Novel devices and interaction techniques for human-scale haptics. The Visual Computer 23, 4 (March 2007), 257-266. https://doi.org/10.1007/s00371-007-0100-4

[43] Frederick Ducatelle, Gianni A. Di Caro, Carlo Pinciroli, and Luca M. Gambardella. 2011. Self-organized cooperation between robotic swarms. Swarm Intelligence 5, 2 (June 2011), 73-96. https://doi.org/10.1007/s11721-011-0053-0

[44] David Escobar-Castillejos, Julieta Noguez, Luis Neri, Alejandra Magana, and Bedrich Benes. 2016. A Review of Simulators with Haptic Devices for Medical Training. Fournal of Medical Systems 40, 4 (April 2016), 1-22. https://doi.org/ 10.1007/s10916-016-0459-8

[45] Cathy Fang, Yang Zhang, Matthew Dworman, and Chris Harrison. 2020. Wireality: Enabling Complex Tangible Geometries in Virtual Reality with Worn Multi-String Haptics. (2020), 10

[46] Martin Feick, Scott Bateman, Anthony Tang, André Miede, and Nicolai Marquardt. 2020. TanGi: Tangible Proxies for Embodied Object Exploration and Manipulation in Virtual Reality. arXiv:2001.03021 [cs] (Jan. 2020). http: //arxiv.org/abs/2001.03021 arXiv: 2001.03021

[47] Daniel Fitzgerald and Hiroshi Ishii. 2018. Mediate: A Spatial Tangible Interface for Mixed Reality. In Extended Abstracts of the 2018 CHI Conference on Human Factors in Computing Systems. ACM, Montreal QC Canada, 1-6. https://doi. org $/ 10.1145 / 3170427.3188472$

[48] Sean Follmer, Daniel Leithinger, Alex Olwal, Akimitsu Hogge, and Hiroshi Ishii. 2013. inFORM: dynamic physical affordances and constraints through shape and object actuation. In Proceedings of the 26th annual ACM symposium on User interface software and technology - UIST '13. ACM Press, St. Andrews, Scotland, United Kingdom, 417-426. https://doi.org/10.1145/2501988.2502032

[49] A. Formaglio, A. Giannitrapani, M. Franzini, D. Prattichizzo, and F. Barbagli. 2005. Performance of Mobile Haptic Interfaces. In Proceedings of the 44th IEEE Conference on Decision and Control. 8343-8348. https://doi.org/10.1109/CDC. 2005.1583513

[50] Ilja Frissen, Jennifer L. Campos, Manish Sreenivasa, and Marc O. Ernst. 2013 Enabling Unconstrained Omnidirectional Walking Through Virtual Environments: An Overview of the CyberWalk Project. In Human Walking in Virtual Environments: Perception, Technology, and Applications, Frank Steinicke, Yon Visell, Jennifer Campos, and Anatole Lécuyer (Eds.). Springer, New York, NY, 113-144. https://doi.org/10.1007/978-1-4419-8432-6 6

[51] Markus Funk, Florian Müller, Marco Fendrich, Megan Shene, Moritz Kolvenbach, Niclas Dobbertin, Sebastian Günther, and Max Mühlhäuser. 2019. Assessing the Accuracy of Point \& Teleport Locomotion with Orientation Indication for Virtual Reality using Curved Trajectories. In Proceedings of the 2019 CHI Conference on Human Factors in Computing Systems - CHI '19. ACM Press, Glasgow, Scotland Uk, 1-12. https://doi.org/10.1145/3290605.3300377

[52] Thomas Galais, Alexandra Delmas, and Rémy Alonso. 2019. Natural interaction in virtual reality: impact on the cognitive load. In Proceedings of the 31st Conference on l'Interaction Homme-Machine Adjunct - IHM '19. ACM Press, Grenoble, France, 1-9. https://doi.org/10.1145/3366551.3370342

[53] Péter Galambos. 2012. Vibrotactile Feedback for Haptics and Telemanipulation: Survey, Concept and Experiment. Acta Polytechnica Hungarica 9, 1 (2012), 25.

[54] Antonio Gomes, Calvin Rubens, Sean Braley, and Roel Vertegaal. 2016. BitDrones: Towards Using 3D Nanocopter Displays as Interactive Self-Levitating Programmable Matter. In Proceedings of the 2016 CHI Conference on Human Factors in Computing Systems - CHI '16. ACM Press, Santa Clara, California, USA, 770-780. https://doi.org/10.1145/2858036.2858519

[55] Eric J. Gonzalez, Parastoo Abtahi, and Sean Follmer. 2020. REACH+: Extending the Reachability of Encountered-type Haptics Devices through Dynamic Redirection in VR. In Proceedings of the 33rd Annual ACM Symposium on User Interface Software and Technology. ACM, Virtual Event USA, 236-248. https://doi.org/10.1145/3379337.3415870

[56] Eric J. Gonzalez and Sean Follmer. 2019. Investigating the Detection of Bimanual Haptic Retargeting in Virtual Reality. In 25th ACM Symposium on Virtual Reality Software and Technology on - VRST '19. ACM Press, Parramatta, NSW, Australia, 1-5. https://doi.org/10.1145/3359996.3364248

[57] Xiaochi Gu, Yifei Zhang, Weize Sun, Yuanzhe Bian, Dao Zhou, and Per Ola Kristensson. 2016. Dexmo: An Inexpensive and Lightweight Mechanical Exoskeleton for Motion Capture and Force Feedback in VR. In Proceedings of the 2016 CHI Conference on Human Factors in Computing Systems - CHI '16. ACM Press, Santa Clara, California, USA, 1991-1995. https://doi.org/10.1145/2858036.2858487

[58] Jan Gugenheimer, Dennis Wolf, Eythor R. Eiriksson, Pattie Maes, and Enrico Rukzio. 2016. GyroVR: Simulating Inertia in Virtual Reality using Head Worn Flywheels. In Proceedings of the 29th Annual Symposium on User Interface Software and Technology. ACM, Tokyo Japan, 227-232. https://doi.org/10.1145/ 2984511.2984535

[59] T. Gutierrez, J. Rodriguez, Y. Velaz, S. Casado, A. Suescun, and E. J. Sanchez. 2010. IMA-VR: A multimodal virtual training system for skills transfer in Industrial Maintenance and Assembly tasks. 19th International Symposium in Robot and Human Interactive Communication (2010). https: //www.academia.edu/15623406/IMA_VR_A_multimodal_virtual_training_ system for_skills transfer in Industrial_Maintenance and Assembly tasks

[60] Sebastian Günther, Dominik Schön, Florian Müller, Max Mühlhäuser, and Martin Schmitz. 2020. PneumoVolley: Pressure-based Haptic Feedback on the Head through Pneumatic Actuation. (2020), 10.

[61] Dustin T. Han, Mohamed Suhail, and Eric D. Ragan. 2018. Evaluating Remapped Physical Reach for Hand Interactions with Passive Haptics in Virtual Reality. IEEE Transactions on Visualization and Computer Graphics 24, 4 (April 2018), 1467-1476. https://doi.org/10.1109/TVCG.2018.2794659

[62] Haption. 2019. Virtuose ${ }^{\mathrm{TM}}$ 6D - HAPTION SA. https://www.haption.com/en/ products-en/virtuose-6d-en.html

[63] Vincent Hayward and Karon Maclean. 2007. Do it yourself haptics: part I. IEEE Robotics \& Automation Magazine 14, 4 (Dec. 2007), 88-104. https://doi.org/10. 1109/M-RA.2007.907921

[64] Zhenyi He, Fengyuan Zhu, Aaron Gaudette, and Ken Perlin. 2017. Robotic Haptic Proxies for Collaborative Virtual Reality. arXiv:1701.08879 [cs] (Jan. 2017). http://arxiv.org/abs/1701.08879 arXiv: 1701.08879

[65] Zhenyi He, Fengyuan Zhu, and Ken Perlin. 2017. PhyShare: Sharing Physical Interaction in Virtual Reality. arXiv:1708.04139 [cs] (Aug. 2017). http://arxiv. org/abs/1708.04139 arXiv: 1708.04139.

[66] Richard M. Held and Nathaniel I. Durlach. 1992. Telepresence. Presence: Teleoperators and Virtual Environments 1, 1 (Jan. 1992), 109-112. https://doi.org/10. 1162/pres.1992.1.1.109

[67] Seongkook Heo, Christina Chung, Geehyuk Lee, and Daniel Wigdor. 2018. Thor's Hammer: An Ungrounded Force Feedback Device Utilizing PropellerInduced Propulsive Force. In Proceedings of the 2018 CHI Conference on Human Factors in Computing Systems - CHI '18. ACM Press, Montreal QC, Canada, 1-11. https://doi.org/10.1145/3173574.3174099

[68] Seongkook Heo, Jaeyeon Lee, and Daniel Wigdor. 2019. PseudoBend: Producing Haptic Illusions of Stretching, Bending, and Twisting Using Grain Vibrations. In Proceedings of the 32nd Annual ACM Symposium on User Interface Software and Technology - UIST '19. ACM Press, New Orleans, LA, USA, 803-813. https: //doi.org/10.1145/3332165.3347941

[69] Anuruddha Hettiarachchi and Daniel Wigdor. 2016. Annexing Reality: Enabling Opportunistic Use of Everyday Objects as Tangible Proxies in Augmented Reality. In Proceedings of the 2016 CHI Conference on Human Factors in Computing Systems - CHI '16. ACM Press, Santa Clara, California, USA, 1957-1967. https: //doi.org/10.1145/2858036.2858134

[70] Matthias Hoppe, Pascal Knierim, Thomas Kosch, Markus Funk, Lauren Futami, Stefan Schneegass, Niels Henze, Albrecht Schmidt, and Tonja Machulla. 2018. VRHapticDrones: Providing Haptics in Virtual Reality through Quadcopters. In Proceedings of the 17th International Conference on Mobile and Ubiquitous Multimedia - MUM 2018. ACM Press, Cairo, Egypt, 7-18. https://doi.org/10. $1145 / 3282894.3282898$

[71] Matthias Hoppe, Daniel Neumann, Stephan Streuber, Albrecht Schmidt, and Tonja-Katrin Machulla. 2020. A Human Touch: Social Touch Increases the Perceived Human-likeness of Agents in Virtual Reality. https://doi.org/10.1145/3313831. 3376719

[72] Hsin-Yu Huang, Chih-Wei Ning, Po-Yao Wang, Jen-Hao Cheng, and Lung-Pan Cheng. 2020. Haptic-go-round: A Surrounding Platform for Encounter-type Haptics in Virtual Reality Experiences. In Proceedings of the 2020 CHI Conference on Human Factors in Computing Systems. ACM, Honolulu HI USA, 1-10. https: //doi.org/10.1145/3313831.3376476

[73] Brent Edward Insko. 2001. Passive Haptics Significantly Enhances Virtual Environments. (2001), 111.

[74] Hiroo Iwata. 2005. CirculaFloor. https://ieeexplore.ieee.org/abstract/document/ 1381227 
[75] Hiroo Iwata. 2013. Locomotion Interfaces. In Human Walking in Virtual Environments: Perception, Technology, and Applications, Frank Steinicke, Yon Visell, Jennifer Campos, and Anatole Lécuyer (Eds.). Springer, New York, NY, 199-219. https://doi.org/10.1007/978-1-4419-8432-6_9

[76] Hiroo Iwata, Hiroaki Yano, Fumitaka Nakaizumi, and Ryo Kawamura. 2001 Project FEELEX: adding haptic surface to graphics. In Proceedings of the 28th annual conference on Computer graphics and interactive techniques - SIGGRAPH '01. ACM Press, Not Known, 469-476. https://doi.org/10.1145/383259.383314

[77] Lynette Jones. 2000. Kinesthetic Sensing. Human and Machine Haptics (2000). http://bdml.stanford.edu/twiki/pub/Haptics/PapersInProgress/jones00.pdf

[78] Erin Kim and Oliver Schneider. 2020. Defining Haptic Experience: Foundations for Understanding, Communicating, and Evaluating HX. In Proceedings of the 2020 CHI Conference on Human Factors in Computing Systems. ACM, Honolulu HI USA, 1-13. https://doi.org/10.1145/3313831.3376280

[79] Lawrence H. Kim, Daniel S. Drew, Veronika Domova, and Sean Follmer. 2020. User-defined Swarm Robot Control. In Proceedings of the 2020 CHI Conference on Human Factors in Computing Systems. ACM, Honolulu HI USA, 1-13. https: //doi.org/10.1145/3313831.3376814

[80] Yaesol Kim, Hyun Jung Kim, and Young J. Kim. 2018. Encountered-type haptic display for large VR environment using per-plane reachability maps Encountered-type Haptic Display for Large VR Environment. Computer Animation and Virtual Worlds 29, 3-4 (May 2018), e1814. https://doi.org/10.1002/cav. 1814

[81] Pascal Knierim, Thomas Kosch, Valentin Schwind, Markus Funk, Francisco Kiss, Stefan Schneegass, and Niels Henze. 2017. Tactile Drones - Providing Immersive Tactile Feedback in Virtual Reality through Quadcopters. In Proceedings of the 2017 CHI Conference Extended Abstracts on Human Factors in Computing Systems - CHI EA '17. ACM Press, Denver, Colorado, USA, 433-436. https: //doi.org/10.1145/3027063.3050426

[82] Luv Kohli. 2010. Redirected touching: Warping space to remap passive haptics In 2010 IEEE Symposium on 3D User Interfaces (3DUI). IEEE, Waltham, MA, USA 129-130. https://doi.org/10.1109/3DUI.2010.5444703

[83] L. Kohli, M. C. Whitton, and F. P. Brooks. 2012. Redirected touching: The effect of warping space on task performance. In 2012 IEEE Symposium on $3 D$ User Interfaces (3DUI). IEEE, Costa Mesa, CA, 105-112. https://doi.org/10.1109/3DUI 2012.6184193

[84] Luv Kohli, Mary C. Whitton, and Frederick P. Brooks. 2013. Redirected Touching Training and adaptation in warped virtual spaces. In 2013 IEEE Symposium on $3 D$ User Interfaces (3DUI). IEEE, Orlando, FL, 79-86. https://doi.org/10.1109/ 3DUI.2013.6550201

[85] Robert Kovacs, Eyal Ofek, Mar Gonzalez Franco, Alexa Fay Siu, Sebastian Marwecki, Christian Holz, and Mike Sinclair. 2020. Haptic PIVOT: On-Demand Handhelds in VR. In Proceedings of the 33rd Annual ACM Symposium on User Interface Software and Technology (UIST '20). Association for Computing Machinery, New York, NY, USA, 1046-1059. https://doi.org/10.1145/3379337.3415854

[86] Mathieu Le Goc, Lawrence H. Kim, Ali Parsaei, Jean-Daniel Fekete, Pierre Dragicevic, and Sean Follmer. 2016. Zooids: Building Blocks for Swarm User Interfaces. In Proceedings of the 29th Annual Symposium on User Interface Software and Technology - UIST '16. ACM Press, Tokyo, Japan, 97-109. https://doi.org/ $10.1145 / 2984511.2984547$

[87] S. J. Lederman and R. L. Klatzky. 2009. Haptic perception: A tutorial. Attention, Perception \& Psychophysics 71, 7 (Oct. 2009), 1439-1459. https://doi.org/10.3758/ APP.71.7.1439

[88] Chaehyun Lee, Min Sik Hong, In Lee, Oh Kyu Choi, Kyung-Lyong Han, Yoo Yeon Kim, Seungmoon Choi, and Jin S Lee. 2007. Mobile Haptic Interface for Large Immersive Virtual Environments: PoMHI v0.5. (2007), 2.

[89] In Lee, Inwook Hwang, Kyung-Lyoung Han, Oh Kyu Choi, Seungmoon Choi, and Jin S. Lee. 2009. System improvements in Mobile Haptic Interface. In World Haptics 2009 - Third Joint EuroHaptics conference and Symposium on Haptic Interfaces for Virtual Environment and Teleoperator Systems. IEEE, Salt Lake City, UT, USA, 109-114. https://doi.org/10.1109/WHC.2009.4810834

[90] Jaeyeon Lee, Mike Sinclair, Mar Gonzalez-Franco, Eyal Ofek, and Christian Holz. 2019. TORC: A Virtual Reality Controller for In-Hand High-Dexterity Finger Interaction. In Proceedings of the 2019 CHI Conference on Human Factors in Computing Systems - CHI '19. ACM Press, Glasgow, Scotland Uk, 1-13. https: //doi.org/10.1145/3290605.3300301

[91] Daniel Leithinger, Sean Follmer, Alex Olwal, Samuel Luescher, Akimitsu Hogge, Jinha Lee, and Hiroshi Ishii. 2013. Sublimate: state-changing virtual and physical rendering to augment interaction with shape displays. In Proceedings of the SIGCHI Conference on Human Factors in Computing Systems - CHI '13. ACM Press, Paris, France, 1441. https://doi.org/10.1145/2470654.2466191

[92] Jean-Claude Lepecq, Lionel Bringoux, Jean-Marie Pergandi, Thelma Coyle, and Daniel Mestre. 2008. Afforded Actions as a Behavioral Assessment of Physical Presence. (2008), 8

[93] Jo-Yu Lo, Da-Yuan Huang, Chen-Kuo Sun, Chu-En Hou, and Bing-Yu Chen. 2018 RollingStone: Using Single Slip Taxel for Enhancing Active Finger Exploration with a Virtual Reality Controller. In The 31st Annual ACM Symposium on User
Interface Software and Technology - UIST '18. ACM Press, Berlin, Germany, 839851. https://doi.org/10.1145/3242587.3242627

[94] Pedro Lopes, Patrik Jonell, and Patrick Baudisch. 2015. Affordance++: Allowing Objects to Communicate Dynamic Use. In Proceedings of the 33rd Annual ACM Conference on Human Factors in Computing Systems - CHI '15. ACM Press, Seoul, Republic of Korea, 2515-2524. https://doi.org/10.1145/2702123.2702128

[95] Pedro Lopes, Sijing You, Lung-Pan Cheng, Sebastian Marwecki, and Patrick Baudisch. 2017. Providing Haptics to Walls \& Heavy Objects in Virtual Reality by Means of Electrical Muscle Stimulation. In Proceedings of the 2017 CHI Conference on Human Factors in Computing Systems - CHI '17. ACM Press, Denver, Colorado, USA, 1471-1482. https://doi.org/10.1145/3025453.3025600

[96] Anatole Lécuyer. 2009. Simulating Haptic Feedback Using Vision: A Survey of Research and Applications of Pseudo-Haptic Feedback. Presence: Teleoperators and Virtual Environments 18, 1 (Feb. 2009), 39-53. https://doi.org/10.1162/pres. 18.1 .39

[97] N. Magnenat-Thalmann, HyungSeok Kim, A. Egges, and S. Garchery. 2005. Believability and Interaction in Virtual Worlds. In 11th International Multimedia Modelling Conference. IEEE, Honolulu, HI, USA, 2-9. https://doi.org/10.1109/ MMMC.2005.24

[98] Lawrence Makin, Gareth Barnaby, and Anne Roudaut. 2019. Tactile and kinesthetic feedbacks improve distance perception in virtual reality. In Proceedings of the 31st Conference on l'Interaction Homme-Machine - IHM '19. ACM Press, Grenoble, France, 1-9. https://doi.org/10.1145/3366550.3372248

[99] Nicolai Marquardt, Miguel A. Nacenta, James E. Young, Sheelagh Carpendale, Saul Greenberg, and Ehud Sharlin. 2009. The Haptic Tabletop Puck: tactile feedback for interactive tabletops. In Proceedings of the ACM International Conference on Interactive Tabletops and Surfaces - ITS '09. ACM Press, Banff, Alberta, Canada, 85. https://doi.org/10.1145/1731903.1731922

[100] Thomas H Massie and J K Salisbury. 1994. The PHANTOM Haptic Interface: A Device for Probing Virtual Objects. (1994), 5.

[101] W. A. McNeely. 1993. Robotic graphics: a new approach to force feedback for virtual reality. In Proceedings of IEEE Virtual Reality Annual International Symposium. 336-341. https://doi.org/10.1109/VRAIS.1993.380761

[102] Leonel Merino, Magdalena Schwarzl, Matthias Kraus, Michael Sedlmair, Dieter Schmalstieg, and Daniel Weiskopf. 2020. Evaluating Mixed and Augmented Reality: A Systematic Literature Review (2009-2019). arXiv:2010.05988 [cs] (Oct. 2020). http://arxiv.org/abs/2010.05988 arXiv: 2010.05988

[103] Judi Moline. 1997. Virtual reality for health care: a survey. Technical Report.

[104] Ken Nakagaki, Artem Dementyev, Sean Follmer, Joseph A. Paradiso, and Hiroshi Ishii. 2016. ChainFORM: A Linear Integrated Modular Hardware System for Shape Changing Interfaces. In Proceedings of the 29th Annual Symposium on User Interface Software and Technology - UIST '16. ACM Press, Tokyo, Japan, 87-96. https://doi.org/10.1145/2984511.2984587

[105] Ken Nakagaki, Luke Vink, Jared Counts, Daniel Windham, Daniel Leithinger, Sean Follmer, and Hiroshi Ishii. 2016. Materiable: Rendering Dynamic Material Properties in Response to Direct Physical Touch with Shape Changing Interfaces. In Proceedings of the 2016 CHI Conference on Human Factors in Computing Systems - CHI '16. ACM Press, Santa Clara, California, USA, 2764-2772. https: //doi.org/10.1145/2858036.2858104

[106] Norbert Nitzsche, Uwe D. Hanebeck, and G. Schmidt. 2003. Design issues of mobile haptic interfaces. Fournal of Robotic Systems 20, 9 (Sept. 2003), 549-556. https://doi.org/10.1002/rob.10105

[107] Donald A. Norman. 2013. The design of everyday things (revised and expanded edition ed.). Basic Books, New York, New York

[108] Optitrack. 2019. Motion Capture Systems. http://optitrack.com/index.html

[109] M. Ortega and S. Coquillart. 2005. Prop-based haptic interaction with co-location and immersion: an automotive application. In IREE International Worksho on Haptic Audio Visual Environments and their Applications, 2005. IEEE, Ottawa, Canada, 23-28. https://doi.org/10.1109/HAVE.2005.1545646

[110] J. Pair, U. Neumann, D. Piepol, and B. Swartout. 2003. FlatWorld: combining Hollywood set-design techniques with VR. IEEE Computer Graphics and Applications 23, 1 (Jan. 2003), 12-15. https://doi.org/10.1109/MCG.2003.1159607

[111] Ryan A. Pavlik, Judy M. Vance, and Greg R. Luecke. 2013. Interacting With a Large Virtual Environment by Combining a Ground-Based Haptic Device and a Mobile Robot Base. In Volume 2B: 33rd Computers and Information in Engineering Conference. ASME, Portland, Oregon, USA, V02BT02A029. https: //doi.org/10.1115/DETC2013-13441

[112] M Poyade, L Molina-Tanco, A Reyes-Lecuona, A Langley, E Frutos, and S Flores. 2012. Validation of a haptic virtual reality simulation in the context of industrial maintenance. (2012), 4

[113] Pragathi Praveena, Daniel Rakita, Bilge Mutlu, and Michael Gleicher. 2020. Supporting Perception of Weight through Motion-induced Sensory Conflicts in Robot Teleoperation. In Proceedings of the 2020 ACM/IEEE International Conference on Human-Robot Interaction. ACM, Cambridge United Kingdom, 509-517. https://doi.org/10.1145/3319502.3374841

[114] William R. Provancher, Mark R. Cutkosky, Katherine J. Kuchenbecker, and Günter Niemeyer. 2005. Contact Location Display for Haptic Perception of Curvature and Object Motion. The International Journal of Robotics Research 24, 
9 (Sept. 2005), 691-702. https://doi.org/10.1177/0278364905057121

[115] Andreas Pusch and Anatole Lécuyer. 2011. Pseudo-haptics: from the theoretical foundations to practical system design guidelines. In Proceedings of the 13th international conference on multimodal interfaces - ICMI '11. ACM Press, Alicante, Spain, 57. https://doi.org/10.1145/2070481.2070494

[116] Susanne Putze, Dmitry Alexandrovsky, Felix Putze, Sebastian Höffner, Jan David Smeddinck, and Rainer Malaka. 2020. Breaking The Experience: Effects of Questionnaires in VR User Studies. In Proceedings of the 2020 CHI Conference on Human Factors in Computing Systems. ACM, Honolulu HI USA, 1-15. https: //doi.org/10.1145/3313831.3376144

[117] Ismo Rakkolainen, Euan Freeman, Antti Sand, Roope Raisamo, and Stephen Brewster. 2020. A Survey of Mid-Air Ultrasound Haptics and Its Applications. IEEE Transactions on Haptics (2020), 1-1. https://doi.org/10.1109/TOH.2020. 3018754

[118] Karan Rangarajan, Heather Davis, and Philip H. Pucher. 2020. Systematic Review of Virtual Haptics in Surgical Simulation: A Valid Educational Tool? fournal of Surgical Education 77, 2 (March 2020), 337-347. https://doi.org/10.1016/j.jsurg. 2019.09.006

[119] Sharif Razzaque, Zachariah Kohn, and Mary C. Whitton. 2001. EUROGRAPHICS 2001 / Jonathan C. Roberts Short Presentation () The Eurographics Association 2001. Redirected Walking.

[120] Michael Rietzler, Florian Geiselhart, Jan Gugenheimer, and Enrico Rukzio. 2018. Breaking the Tracking: Enabling Weight Perception using Perceivable Tracking Offsets. In Proceedings of the 2018 CHI Conference on Human Factors in Computing Systems - CHI '18. ACM Press, Montreal QC, Canada, 1-12. https://doi.org/10. $1145 / 3173574.3173702$

[121] Michael Rietzler, Gabriel Haas, Thomas Dreja, Florian Geiselhart, and Enrico Rukzio. 2019. Virtual Muscle Force: Communicating Kinesthetic Forces Through Pseudo-Haptic Feedback and Muscle Input. In Proceedings of the 32nd Annual ACM Symposium on User Interface Software and Technology - UIST '19. ACM Press, New Orleans, LA, USA, 913-922. https://doi.org/10.1145/3332165.3347871

[122] Calvin Rubens, Sean Braley, Antonio Gomes, Daniel Goc, Xujing Zhang, Juan Pablo Carrascal, and Roel Vertegaal. 2015. BitDrones: Towards Levitating Programmable Matter Using Interactive 3D Quadcopter Displays. In Proceedings of the 28th Annual ACM Symposium on User Interface Software \& Technology - UIST '15 Adjunct. ACM Press, Daegu, Kyungpook, Republic of Korea, 57-58. https://doi.org/10.1145/2815585.2817810

[123] K. Martin Sagayam and D. Jude Hemanth. 2017. Hand posture and gesture recognition techniques for virtual reality applications: a survey. Virtual Reality 21, 2 (June 2017), 91-107. https://doi.org/10.1007/s10055-016-0301-0

[124] Shahabedin Sagheb, Frank Wencheng Liu, Alireza Bahremand, Assegid Kidane, and Robert LiKamWa. 2019. SWISH: A Shifting-Weight Interface of Simulated Hydrodynamics for Haptic Perception of Virtual Fluid Vessels. In Proceedings of the 32nd Annual ACM Symposium on User Interface Software and Technology UIST '19. ACM Press, New Orleans, LA, USA, 751-761. https://doi.org/10.1145/ 3332165.3347870

[125] Majed Samad, Elia Gatti, Anne Hermes, Hrvoje Benko, and Cesare Parise. 2019. Pseudo-Haptic Weight: Changing the Perceived Weight of Virtual Objects By Manipulating Control-Display Ratio. In Proceedings of the 2019 CHI Conference on Human Factors in Computing Systems - CHI '19. ACM Press, Glasgow, Scotland Uk, 1-13. https://doi.org/10.1145/3290605.3300550

[126] Massimo Satler, Carlo A. Avizzano, and Emanuele Ruffaldi. 2011. Control of a desktop mobile haptic interface. In 2011 IEEE World Haptics Conference. IEEE, Istanbul, 415-420. https://doi.org/10.1109/WHC.2011.5945522

[127] M. Sato. 2002. SPIDAR and virtual reality. In Proceedings of the 5th Biannual World Automation Congress, Vol. 13. 17-23. https://doi.org/10.1109/WAC.2002.1049515

[128] Gian-Luca Savino. 2020. Virtual Smartphone: High Fidelity Interaction with Proxy Objects in Virtual Reality. arXiv:2010.00942 [cs] (Oct. 2020). http://arxiv. org/abs/2010.00942 arXiv: 2010.00942.

[129] Dominik Schmidt, Rob Kovacs, Vikram Mehta, Udayan Umapathi, Sven Köhler, Lung-Pan Cheng, and Patrick Baudisch. 2015. Level-Ups: Motorized Stilts that Simulate Stair Steps in Virtual Reality. In Proceedings of the 33rd Annual ACM Conference on Human Factors in Computing Systems - CHI '15. ACM Press, Seoul, Republic of Korea, 2157-2160. https://doi.org/10.1145/2702123.2702253

[130] Martijn J. Schuemie, Peter van der Straaten, Merel Krijn, and Charles A.P.G. van der Mast. 2001. Research on Presence in Virtual Reality: A Survey. $C y$ berPsychology \& Behavior 4, 2 (April 2001), 183-201. https://doi.org/10.1089/ 109493101300117884

[131] Valentin Schwind, Pascal Knierim, Nico Haas, and Niels Henze. 2019. Using Presence Questionnaires in Virtual Reality. In Proceedings of the 2019 CHI Con ference on Human Factors in Computing Systems - CHI '19. ACM Press, Glasgow, Scotland Uk, 1-12. https://doi.org/10.1145/3290605.3300590

[132] Hasti Seifi, Farimah Fazlollahi, Michael Oppermann, John Andrew Sastrillo, Jessica Ip, Ashutosh Agrawal, Gunhyuk Park, Katherine J. Kuchenbecker, and Karon E. MacLean. 2019. Haptipedia: Accelerating Haptic Device Discovery to Support Interaction \& Engineering Design. In Proceedings of the $2019 \mathrm{CHI}$ Conference on Human Factors in Computing Systems - CHI '19. ACM Press, Glasgow, Scotland Uk, 1-12. https://doi.org/10.1145/3290605.3300788
[133] Emily Shaw, Tessa Roper, Tommy Nilsson, Glyn Lawson, Sue V. G. Cobb, and Daniel Miller. 2019. The Heat is On: Exploring User Behaviour in a Multisensory Virtual Environment for Fire Evacuation. Proceedings of the 2019 CHI Conference on Human Factors in Computing Systems - CHI '19 (2019), 1-13. https://doi.org/ 10.1145/3290605.3300856 arXiv: 1902.04573.

[134] Jotaro Shigeyama, Takeru Hashimoto, Shigeo Yoshida, Takuji Narumi, Tomohiro Tanikawa, and Michitaka Hirose. 2019. Transcalibur: A Weight Shifting Virtual Reality Controller for 2D Shape Rendering based on Computational Perception Model. In Proceedings of the 2019 CHI Conference on Human Factors in Computing Systems - CHI '19. ACM Press, Glasgow, Scotland Uk, 1-11. https://doi.org/10. $1145 / 3290605.3300241$

[135] Adalberto L. Simeone, Eduardo Velloso, and Hans Gellersen. 2015. Substitutional Reality: Using the Physical Environment to Design Virtual Reality Experiences. In Proceedings of the 33rd Annual ACM Conference on Human Factors in Computing Systems - CHI '15. ACM Press, Seoul, Republic of Korea, 3307-3316. https://doi.org/10.1145/2702123.2702389

[136] Mike Sinclair, Eyal Ofek, Mar Gonzalez-Franco, and Christian Holz. 2019. CapstanCrunch: A Haptic VR Controller with User-supplied Force Feedback. In Proceedings of the 32nd Annual ACM Symposium on User Interface Software and Technology - UIST '19. ACM Press, New Orleans, LA, USA, 815-829. https://doi.org/10.1145/3332165.3347891

[137] Mike Sinclair, Michel Pahud, and Hrvoje Benko. 2014. TouchMover 2.0 - 3D touchscreen with force feedback and haptic texture. In 2014 IEEE Haptics Symposium (HAPTICS). IEEE, Houston, TX, USA, 1-6. https://doi.org/10.1109/ HAPTICS.2014.6775425

[138] Alexa F. Siu, Eric J. Gonzalez, Shenli Yuan, Jason B. Ginsberg, and Sean Follmer. 2018. shapeShift: 2D Spatial Manipulation and Self-Actuation of Tabletop Shape Displays for Tangible and Haptic Interaction. In Proceedings of the $2018 \mathrm{CHI}$ Conference on Human Factors in Computing Systems - CHI '18. ACM Press, Montreal QC, Canada, 1-13. https://doi.org/10.1145/3173574.3173865

[139] Mel Slater. 1999. Measuring Presence: A Response to the Witmer and Singer Presence Questionnaire. Presence: Teleoperators and Virtual Environments 8, 5 (Oct. 1999), 560-565. https://doi.org/10.1162/105474699566477 Publisher: MIT Press.

[140] Mel Slater, Martin Usoh, and Anthony Steed. 1994. Depth of Presence in Virtual Environments. Presence: Teleoperators and Virtual Environments 3, 2 (Jan. 1994), 130-144. https://doi.org/10.1162/pres.1994.3.2.130

[141] Anthony Steed, Sebastian Friston, Vijay Pawar, and David Swapp. 2020. Docking Haptics: Extending the Reach of Haptics by Dynamic Combinations of Grounded and Worn Devices. arXiv:2002.06093 [cs] (Feb. 2020). http://arxiv.org/abs/2002. 06093 arXiv: 2002.06093

[142] Frank Steinicke, Visell Yon, Jennifer Campos, and Anatole Lecuyer (Eds.). 2013. Human walking in virtual environments: perception, technology, and applications. Springer, New York,NY. OCLC: 856865949.

[143] Patrick L. Strandholt, Oana A. Dogaru, Niels C. Nilsson, Rolf Nordahl, and Stefania Serafin. 2020. Knock on Wood: Combining Redirected Touching and Physical Props for Tool-Based Interaction in Virtual Reality. In Proceedings of the 2020 CHI Conference on Human Factors in Computing Systems. ACM, Honolulu HI USA, 1-13. https://doi.org/10.1145/3313831.3376303

[144] Evan Strasnick, Christian Holz, Eyal Ofek, Mike Sinclair, and Hrvoje Benko. 2018. Haptic Links: Bimanual Haptics for Virtual Reality Using Variable Stiffness Actuation. In Proceedings of the 2018 CHI Conference on Human Factors in Computing Systems - CHI '18. ACM Press, Montreal QC, Canada, 1-12. https://doi.org/10.1145/3173574.3174218

[145] Paul Strohmeier, Seref Güngör, Luis Herres, Dennis Gudea, Bruno Fruchard, and Jürgen Steimle. 2020. bARefoot: Generating Virtual Materials using Motion Coupled Vibration in Shoes. In Proceedings of the 33rd Annual ACM Symposium on User Interface Software and Technology. ACM, Virtual Event USA, 579-593. https://doi.org/10.1145/3379337.3415828

[146] Yuqian Sun, Shigeo Yoshida, Takuji Narumi, and Michitaka Hirose. 2019. PaCaPa: A Handheld VR Device for Rendering Size, Shape, and Stiffness of Virtual Objects in Tool-based Interactions. In Proceedings of the 2019 CHI Conference on Human Factors in Computing Systems - CHI '19. ACM Press, Glasgow, Scotland Uk, 1-12. https://doi.org/10.1145/3290605.3300682

[147] Ivan Sutherland. 1965. The Ultimate Display. (1965), 2.

[148] Ryo Suzuki, Hooman Hedayati, Clement Zheng, James Bohn, Daniel Szafir, Ellen Yi-Luen Do, Mark D Gross, and Daniel Leithinger. 2020. RoomShift: Room-scale Dynamic Haptics for VR with Furniture-moving Swarm Robots. (2020), 11.

[149] Ryo Suzuki, Junichi Yamaoka, Daniel Leithinger, Tom Yeh, Mark D. Gross, Yoshihiro Kawahara, and Yasuaki Kakehi. 2018. Dynablock: Dynamic 3D Printing for Instant and Reconstructable Shape Formation. In The 31st Annual ACM Symposium on User Interface Software and Technology - UIST '18. ACM Press, Berlin, Germany, 99-111. https://doi.org/10.1145/3242587.3242659

[150] Ryo Suzuki, Clement Zheng, Yasuaki Kakehi, Tom Yeh, Ellen Yi-Luen Do, Mark D Gross, and Daniel Leithinger. 2019. ShapeBots: Shape-changing Swarm Robots. (2019), 13.

[151] N. Takizawa, H. Yano, H. Iwata, Y. Oshiro, and N. Ohkohchi. 2017. EncounteredType Haptic Interface for Representation of Shape and Rigidity of 3D Virtual 
Objects. IEEE Transactions on Haptics 10, 4 (Oct. 2017), 500-510, https://doi. org/10.1109/TOH.2017.2740934

[152] Anthony Talvas, Maud Marchal, and Anatole Lecuyer. 2014. A Survey on Bimanual Haptic Interaction. IEEE Transactions on Haptics 7, 3 (July 2014), 285-300. https://doi.org/10.1109/TOH.2014.2314456

[153] Shan-Yuan Teng, Tzu-Sheng Kuo, Chi Wang, Chi-huan Chiang, Da-Yuan Huang, Liwei Chan, and Bing-Yu Chen. 2018. PuPoP: Pop-up Prop on Palm for Virtual Reality. In The 31st Annual ACM Symposium on User Interface Software and Technology - UIST '18. ACM Press, Berlin, Germany, 5-17. https://doi.org/10. $1145 / 3242587.3242628$

[154] Shan-Yuan Teng, Cheng-Lung Lin, Chi-huan Chiang, Tzu-Sheng Kuo, Liwe Chan, Da-Yuan Huang, and Bing-Yu Chen. 2019. TilePoP: Tile-type Pop-up Prop for Virtual Reality. (2019), 11

[155] Marc Teyssier, Gilles Bailly, Catherine Pelachaud, and Eric Lecolinet. 2020. Conveying Emotions Through Device-Initiated Touch. IEEE Transactions on Affective Computing (2020), 1-1. https://doi.org/10.1109/TAFFC.2020.3008693

[156] N. G. Tsagarakis, T. Horne, and D. G. Caldwell. 2005. SLIP AESTHEASIS: a portable 2D slip/skin stretch display for the fingertip. In First foint Eurohaptics Conference and Symposium on Haptic Interfaces for Virtual Environment and Teleoperator Systems. World Haptics Conference. 214-219. https://doi.org/10. 1109/WHC.2005.117

[157] Hsin-Ruey Tsai and Bing-Yu Chen. 2019. ElastImpact: 2.5D Multilevel Instant Impact Using Elasticity on Head-Mounted Displays. In Proceedings of the 32nd Annual ACM Symposium on User Interface Software and Technology. ACM, New Orleans LA USA, 429-437. https://doi.org/10.1145/3332165.3347931

[158] Hsin-Ruey Tsai and Jun Rekimoto. 2018. ElasticVR: Providing Multi-level Active and Passive Force Feedback in Virtual Reality Using Elasticity. In Extended Abstracts of the 2018 CHI Conference on Human Factors in Computing Systems - CHI '18. ACM Press, Montreal QC, Canada, 1-4. https://doi.org/10.1145/ 3170427.3186540

[159] Evgeny Tsykunov, Roman Ibrahimov, Derek Vasquez, and Dzmitry Tsetserukou 2019. SlingDrone: Mixed Reality System for Pointing and Interaction Using a Single Drone. In 25th ACM Symposium on Virtual Reality Software and Technology on - VRST '19. ACM Press, Parramatta, NSW, Australia, 1-5. https://doi.org/10. $1145 / 3359996.3364271$

[160] Evgeny Tsykunov and Dzmitry Tsetserukou. 2019. WiredSwarm: High Resolution Haptic Feedback Provided by a Swarm of Drones to the User's Fingers for VR interaction. In 25th ACM Symposium on Virtual Reality Software and Technology on - VRST '19. ACM Press, Parramatta, NSW, Australia, 1-2. https://doi.org/10.1145/3359996.3364789

[161] Sebastian Ullrich. 2012. Haptic Palpation for Medical Simulation in Virtual Environments. IEEE TRANSACTIONS ON VISUALIZATION AND COMPUTER GRAPHICS 18, 4 (2012), 9.

[162] Martin Usoh, Kevin Arthur, Mary C. Whitton, Rui Bastos, Anthony Steed, Mel Slater, and Frederick P. Brooks. 1999. Walking > walking-in-place > flying, in virtual environments. In Proceedings of the 26th annual conference on Computer graphics and interactive techniques - SIGGRAPH '99. ACM Press, Not Known, 359-364. https://doi.org/10.1145/311535.311589

[163] Martin Usoh, Ernest Catena, Sima Arman, and Mel Slater. 2000. Using Presence Questionnaires in Reality. Presence: Teleoperators and Virtual Environments 9, 5 (Oct. 2000), 497-503. https://doi.org/10.1162/105474600566989

[164] Varalakshmi, Thriveni, Venugopal, and Patnaik. 2012. Haptics: State of the Art Survey. IFCSI International Fournal of Computer Science Issues (2012). https: //core.ac.uk/download/pdf/25725449.pdf

[165] David Steeven Villa Salazar, Claudio Pacchierotti, Xavier De Tinguy De La Girouliere, Anderson Maciel, and Maud Marchal. 2020. Altering the Stiffness, Friction, and Shape Perception of Tangible Objects in Virtual Reality Using Wearable Haptics. IEEE Transactions on Haptics (2020), 1-1. https: //doi.org/10.1109/TOH.2020.2967389

[166] Emanuel Vonach, Clemens Gatterer, and Hannes Kaufmann. 2017. VRRobot: Robot actuated props in an infinite virtual environment. In 2017 IEEE Virtual Reality (VR). IEEE, Los Angeles, CA, USA, 74-83. https://doi.org/10.1109/VR. 2017.7892233

[167] Chi Wang, Da-Yuan Huang, Shuo-Wen Hsu, Cheng-Lung Lin, Yeu-Luen Chiu, Chu-En Hou, and Bing-Yu Chen. 2020. Gaiters: Exploring Skin Stretch Feedback on the Legs for Enhancing Virtual Reality Experiences. (2020), 14.

[168] Dangxiao Wang, Yuan Guo, Zhang Yuru, XY Weiliang, and WWIA Jing. 2020 Haptic display for virtual reality: progress and challenges | Elsevier Enhanced Reader. https://doi.org/10.3724/SP.J.2096-5796.2019.0008 ISSN: 2096-5796.

[169] Dangxiao Wang, Kouhei Ohnishi, and Weiliang Xu. 2020. Multimodal Haptic Display for Virtual Reality: A Survey. IEEE Transactions on Industrial Electronics 67, 1 (Jan. 2020), 610-623. https://doi.org/10.1109/TIE.2019.2920602

[170] Yuntao Wang, Hanchuan Li, Zhengyi Cao, Huiyi Luo, Ke Ou, John Raiti, Chun Yu, Shwetak Patel, and Yuanchun Shi. 2020. MoveVR: Enabling Multiform Force Feedback in Virtual Reality using Household Cleaning Robot. (2020), 12.

[171] Tzu-Yun Wei, Hsin-Ruey Tsai, Yu-So Liao, Chieh Tsai, Yi-Shan Chen, Chi Wang, and Bing-Yu Chen. 2020. ElastiLinks: Force Feedback between VR Controllers with Dynamic Points of Application of Force. In Proceedings of the 33rd Annual
ACM Symposium on User Interface Software and Technology. ACM, Virtual Event USA, 1023-1034. https://doi.org/10.1145/3379337.3415836

[172] Alan Wexelblat. 1993. Virtual reality: applications and explorations. http://libertar.io/lab/wp-content/uploads/2016/02/Virtual.Reality.Applications.And_.Explorations.pdf/page $=164$ Myron Krueger, Artificial reality 2 An easy entry to Virtual reality Chap 7.

[173] Eric Whitmire, Hrvoje Benko, Christian Holz, Eyal Ofek, and Mike Sinclair. 2018. Haptic Revolver: Touch, Shear, Texture, and Shape Rendering on a Reconfigurable Virtual Reality Controller. In Proceedings of the 2018 CHI Conference on Human Factors in Computing Systems - CHI '18. ACM Press, Montreal QC, Canada, 1-12. https://doi.org/10.1145/3173574.3173660

[174] Frederik Winther, Linoj Ravindran, Kasper Paabol Svendsen, and Tiare Feuchtner. 2020. Design and Evaluation of a VR Training Simulation for Pump Maintenance Based on a Use Case at Grundfos. In 2020 IEEE Conference on Virtual Reality and 3D User Interfaces (VR). IEEE, Atlanta, GA, USA, 738-746. https://doi.org/10.1109/VR46266.2020.1580939036664

[175] Bob G. Witmer and Michael J. Singer. 1998. Measuring Presence in Virtual Environments: A Presence Questionnaire. Presence: Teleoperators and Virtual Environments 7, 3 (June 1998), 225-240. https://doi.org/10.1162/105474698565686

[176] Haijun Xia, Sebastian Herscher, Ken Perlin, and Daniel Wigdor. 2018. Spacetime: Enabling Fluid Individual and Collaborative Editing in Virtual Reality. In The 31st Annual ACM Symposium on User Interface Software and Technology - UIST '18. ACM Press, Berlin, Germany, 853-866. https://doi.org/10.1145/3242587.3242597

[177] Pingjun Xia. 2016. Haptics for Product Design and Manufacturing Simulation. IEEE Transactions on Haptics 9, 3 (July 2016), 358-375. https://doi.org/10.1109/ TOH.2016.2554551

[178] Kotaro Yamaguchi, Ginga Kato, Yoshihiro Kuroda, Kiyoshi Kiyokawa, and Haruo Takemura. 2016. A Non-grounded and Encountered-type Haptic Display Using a Drone. In Proceedings of the 2016 Symposium on Spatial User Interaction - SUI '16. ACM Press, Tokyo, Japan, 43-46. https://doi.org/10.1145/2983310.2985746

[179] Jackie (Junrui) Yang, Christian Holz, Eyal Ofek, and Andrew D. Wilson. 2019. DreamWalker: Substituting Real-World Walking Experiences with a Virtual Reality. In Proceedings of the 32nd Annual ACM Symposium on User Interface Software and Technology - UIST '19. ACM Press, New Orleans, LA, USA, 10931107. https://doi.org/10.1145/3332165.3347875

[180] Yuan-Syun Ye, Hsin-Yu Chen, and Liwei Chan. 2019. Pull-Ups: Enhancing Suspension Activities in Virtual Reality with Body-Scale Kinesthetic Force Feedback. In Proceedings of the 32nd Annual ACM Symposium on User Interface Software and Technology - UIST '19. ACM Press, New Orleans, LA, USA, 791-801. https://doi.org/10.1145/3332165.3347874

[181] Yan Yixian, Kazuki Takashima, Anthony Tang, Takayuki Tanno, Kazuyuki Fujita, and Yoshifumi Kitamura. 2020. ZoomWalls: Dynamic Walls that Simulate Haptic Infrastructure for Room-scale VR World. In Proceedings of the 33rd Annual ACM Symposium on User Interface Software and Technology (UIST '20). Association for Computing Machinery, New York, NY, USA, 223-235. https://doi.org/10. 1145/3379337.3415859

[182] Yasuyoshi Yokokohji, Ralph L. Hollis, and Takeo Kanade. 1999. WYSIWYF Display: A Visual/Haptic Interface to Virtual Environment. Presence: Teleoperators and Virtual Environments 8, 4 (Aug. 1999), 412-434. https://doi.org/10.1162/ 105474699566314

[183] Y. Yokokohji, J. Kinoshita, and T. Yoshikawa. 2001. Path planning for encountered-type haptic devices that render multiple objects in 3D space. In Proceedings IEEE Virtual Reality 2001. 271-278. https://doi.org/10.1109/VR.2001. 913796

[184] Yasuyoshi Yokokohji, Nobuhiko Muramori, Yuji Sato, and Tsuneo Yoshikawa. 2005. Haptic Display for Multiple Fingertip Contacts Based on the Observation of Human Grasping Behaviors.

[185] Shigeo Yoshida, Yuqian Sun, and Hideaki Kuzuoka. 2020. PoCoPo: Handheld Pin-based Shape Display for Haptic Rendering in Virtual Reality. In Proceedings of the 2020 CHI Conference on Human Factors in Computing Systems. ACM, Honolulu HI USA, 1-13. https://doi.org/10.1145/3313831.3376358

[186] Andre Zenner and Antonio Kruger. 2017. Shifty: A Weight-Shifting Dynamic Passive Haptic Proxy to Enhance Object Perception in Virtual Reality. IEEE Transactions on Visualization and Computer Graphics 23, 4 (April 2017), 12851294. https://doi.org/10.1109/TVCG.2017.2656978

[187] André Zenner and Antonio Krüger. 2019. Drag:on: A Virtual Reality Controller Providing Haptic Feedback Based on Drag and Weight Shift. In Proceedings of the 2019 CHI Conference on Human Factors in Computing Systems - CHI '19. ACM Press, Glasgow, Scotland Uk, 1-12. https://doi.org/10.1145/3290605.3300441

[188] QinPing Zhao. 2009. A survey on virtual reality. Science in China Series F: Information Sciences 52, 3 (March 2009), 348-400. https://doi.org/10.1007/s11432009-0066-0

[189] Yiwei Zhao and Sean Follmer. 2018. A Functional Optimization Based Approach for Continuous 3D Retargeted Touch of Arbitrary, Complex Boundaries in Haptic Virtual Reality. In Proceedings of the 2018 CHI Conference on Human Factors in Computing Systems - CHI '18. ACM Press, Montreal QC, Canada, 1-12. https://doi.org/10.1145/3173574.3174118 
[190] Yiwei Zhao, Lawrence H. Kim, Ye Wang, Mathieu Le Goc, and Sean Follmer 2017. Robotic Assembly of Haptic Proxy Objects for TangibleInteraction and Virtual Reality. In Proceedings of the Interactive Surfaces and Spaces on ZZZ ISS '17. ACM Press, Brighton, United Kingdom, 82-91. https://doi.org/10.1145 3132272.3134143

[191] Ning-Ning Zhou and Yu-Long Deng. 2009. Virtual reality: A state-of-the-art survey. International fournal of Automation and Computing 6, 4 (Nov. 2009) 319-325. https://doi.org/10.1007/s11633-009-0319-9

[192] Mounia Ziat, Taylor Rolison, Andrew Shirtz, Daniel Wilbern, and Carrie Anne Balcer. 2014. Enhancing virtual immersion through tactile feedback. In Proceedings of the adjunct publication of the 27th annual ACM symposium on User interface software and technology - UIST'14 Adjunct. ACM Press, Honolulu, Hawaii,
USA, 65-66. https://doi.org/10.1145/2658779.2659116

[193] Daniel Zielasko and Bernhard E Riecke. 2020. Either Give Me a Reason to Stand or an Opportunity to Sit in VR. (2020), 3.

[194] C. B. Zilles and J. K. Salisbury. 1995. A constraint-based god-object method for haptic display. In In International Conference on Intelligent Robots and Systems. 146-151.

[195] Peter Zimmermann. 2008. Virtual Reality Aided Design. A survey of the use of VR in automotive industry. (Jan. 2008). https://doi.org/10.1007/978-1-40208200-9_13 